\title{
Mechanics of High-Flexible Beams under Live Loads
}

\author{
Luca Lanzoni $^{1}$, Angelo Marcello Tarantino ${ }^{2}$
}

November 22, 2019

${ }^{1}$ DIEF, Università di Modena e Reggio Emilia, via P. Vivarelli 10, 41125, Modena, Italy, e-mail: luca.lanzoni@unimore.it,

${ }^{2}$ DIEF, Università di Modena e Reggio Emilia, via P. Vivarelli 10, 41125, Modena, Italy, e-mail: angelomarcello.tarantino@unimore.it

\begin{abstract}
In this paper the mathematical formulation of the equilibrium problem of high-flexible beams in the framework of fully nonlinear structural mechanics is presented. The analysis is based on the recent model proposed by L. Lanzoni and A.M. Tarantino: The bending of beams in finite elasticity. J. Elasticity (2019) DOI: 10.1007/s10659-019-09746-8 [1]. In this model the complete three-dimensional kinematics of the beam is taken into account, both deformations and displacements are considered large and a nonlinear constitutive law in assumed. After having illustrated and discussed the peculiar mechanical aspects of this special class of structures, the criteria and methods of analysis have been addressed. A classification of the structures based on the degree of kinematic constraints has been proposed, distinguishing between isogeometric and hypergeometric structures. External static loads dependent on deformation (live loads) are also considered. The governing equations are derived on the basis of a moment-curvature relationship obtained in [1]. The governing equations take the form of a highly nonlinear coupled system of equations in integral form, which is solved through an iterative numerical procedure. Finally, the proposed analysis is applied to some popular structural systems subjected to dead and live loads. The results are compared and discussed.
\end{abstract}

Keywords: Finite elasticity; Hyperelasticity; Equilibrium; Highflexible beam; Live loads; Bending moment.

\section{Introduction}

During the period from the end of the seventeenth century to the second half of the eighteenth century the theory of elastic curves was one of the most studied 
themes, sometimes not without controversy and rivalry, by the major exponents of mathematical and mechanical culture.

As a result of its important developments in the analysis of elastic structures, the determination of the curvature of an inflexed beam was undoubtedly the topic of principal interest. The basic equation that relates curvature and bending moment was first treated by Jacob Bernoulli [2], who postulated a law of proportionality between these two functions. Later on, by following a suggestion made by Daniel Bernoulli [3], L. Euler perfected the differential equation of the deflection curve and proceeded with the solution of various problems of the Elastica [4], [5]. J.L. Lagrange was the first to study the Elastica considering a uniform thin column subjected to a vertical concentrated load at its upper end [6]. Although the Elastica equation was obtained at the end of the seventeenth century, its deflection curves had not been accurately plotted until Max Born's $1906 \mathrm{PhD}$ thesis, entitled: Investigations on the stability of the elastic line in plane and space, under different boundary conditions [7]. In essence, the Elastica was conceived as an ideal thin lamina capable of exhibiting large displacements. Namely, a geometrically simplified structural model able to grasp only some nonlinear aspects of the general equilibrium problem.

Successively, many mathematicians and engineers researched the Elastica theory. The case of a horizontal Elastica with one end clamped, the other free and a vertical load applied to the free end was investigated by Barten [8], [9] and Bisshopf and Drucker [10]. The Elastica subjected to a uniformly distributed load was approximately treated by Rohde [11]. Frisch-Fay [12] analyzed the case in which loading is modeled by a system of concentrated loads. Wang et al. [13] presented a series-form solution for the case treated by Barten. In these works, the more widely used solution methods include power series, complete and incomplete elliptic integrals, numerical procedures using the fourth order Runge-Kutta method. Within the numerical methods, also many FEM analyses have been carried out [14], [15].

The scientific interest for the Elastica theory has never diminished, even today numerous papers can be found in the Literature (see, for example, [16], [17] and [18]). In addition, the Elastica has been used to simulate a wide range of new practical problems (see, for example, [19], [20], [21], [22], [23] and [24]). The state of the art of the Elastica theory can be found in the fundamental works by Bigoni [25] and O'Reilly [26].

The mathematical formulation of the Elastica model is based on three fundamental hypotheses: The displacements are considered large, the deformations small and the classic constitutive law of the elastic linear theory is taken into account. In these circumstances, the nonlinearities of the governing equation are due solely to large displacements, which require to impose the equilibrium conditions with respect to the deformed configuration. Therefore the theoretical treatment of the Elastica remains somewhat hybrid, halfway between linear and nonlinear theory. Furthermore, the Elastica model is one-dimensional and does not fully describe the three-dimensional behavior of an inflexed beam.

The equilibrium problem of inflexed hyperelastic blocks and short beams has been investigated by Lanzoni and Tarantino [27] in the fully nonlinear context of 
the finite elasticity. Following a semi-inverse approach, a three-dimensional kinematic model, where the longitudinal bending is accompanied by the transversal anticlastic deformation of cross-sections, has been formulated. This kinematic model is quite different from the others existing in the Literature, where the bending of a solid was essentially formulated for a two-dimensional context, neglecting systematically the deformation of the cross sections [28]. In [27], explicit formulae, which describe the displacement field, stretches and stresses at each point of the beam using both the Lagrangian and Eulerian descriptions, have been obtained.

The analysis was then extended to the class of slender beams inflexed under constant curvature [29], adopting the following hypotheses: During longitudinal bending, cross sections maintain their planarity and are inflexed in their plane with constant anticlastic curvature. These hypotheses, that underlie the theoretical formulation, have subsequently been verified through a numerical analysis and an experimental campaign. The numerical analysis is based on the finite element method (FEM), whereas a test equipment prototype has been designed and manufactured for the large bending of slender beams [29], [30].

The beam model proposed in [27] for the uniform inflexion was then developed to study the general case with variable curvature [1]. Such generalization allowed to derive a completely nonlinear moment-curvature relationship for the beam axis. This relationship has a fairly complex expression. However, for the class of slender beams, it has been shown in [1] that, for a wide range of curvatures, the relationship can be simplified by assuming a law of proportionality between the external bending moment and the curvature itself. This linearity is lost due to high curvatures, such as when the beam is inflexed until to form a tight loop, or when particular constitutive laws, characterized by a strong nonlinear behavior already for low stress values, are adopted. In the conditions specified above, the axis of an inflexed beam in the context of the general theory is bent according to a law very similar to that of Elastica. Thus, although the structural models considered are different, the study carried out in [1] constitutes an indirect demonstration of validity of the celebrated hypothesis of Jacob Bernoulli [2]. Furthermore, the applicability limits of this hypothesis are also established.

Using the beam model presented in [1], the equilibrium problem for highflexible structures is investigated in this paper. After having illustrated and discussed the peculiar mechanical aspects of high-flexible structures, a method of analysis is presented. A classification of the structures on the basis of the degree of kinematic constraints has been proposed, distinguishing between isogeometric and hypergeometric structures. In the problem formulation, external static loads dependent on deformation (live loads) are also considered. The governing equations for the equilibrium of high-flexible structures take the form of a highly nonlinear coupled system of equations in integral form, which is solved by an iterative numerical procedure.

The paper is organized as follows. In Section 2, some basic equations, strictly necessary to set the problem formulation, are recalled. In particular, the moment-curvature relationship derived in [1] is discussed. The static external 
actions are classified in dead and live loads in Section 3, where a mathematical description is provided to facilitate their assignment in the problem formulation. In Section 4, the nonlinear equilibrium problem for isogeometric beams is formulated. Successively, some applications are performed considering two typical structural systems: The cantilever beam and the simply supported beam. Both structures are subjected to uniformly distributed dead and live loads. The live loads are such as to always remain orthogonal to the deformed axis of the beam. For each single example treated, the shape assumed by the beam in the deformed configuration and the diagrams of the normal force, shear force and bending moment are assessed as the load multiplier increases. Stretches and stresses in the cross sections, using both the Lagrangian and Eulerian descriptions, are also computed. Likewise, the analysis is developed for hypergeometric beams in Section 5. After extending the problem formulation, a beam clamped at one end and supported at the other, subjected to both dead and live loads, is studied. Finally, the solutions obtained were verified numerically in Section 6 by checking that the local equilibrium equations are satisfied at each point of the beam, namely that the material divergence of the Piola-Kirchhoff stress tensor is practically zero everywhere. The Conclusions, delivered in Section 7, close the paper.

\section{Basic equations}

A hyperelastic body $\overline{\mathcal{B}}$ composed of a homogeneous, isotropic and compressible material, having the shape of a rectangular parallelepiped, is considered. The symbols $B, H$ and $L$ are used to indicate the width, height and length of the body, respectively. As is typical in the case of slender beams, the length $L$ is predominant on both transverse dimensions $B$ and $H$. Reference is made to a material system of Cartesian coordinates $\{O, X, Y, Z\}$, as shown in Fig. 1a.

In [1], the equilibrium problem for inflexed slender beams has been formulated in the fully nonlinear context of finite elasticity. Specifically, no hypothesis of smallness for the deformation and displacement fields has been introduced and the constitutive law has been assumed to be nonlinear. The three-dimensionality of the problem is maintained, without renouncing to study none of the three components of the displacement field. The kinematic model adopted has been based on Bernoulli-Navier hypothesis. This assumption, which is very popular in the linear theory of beams under pure bending, predicts the conservation of the planarity of cross sections after the deformation. According to the anticlastic effect, also the cross sections are considered inflexed in their plane. The reliability of these hypotheses has been checked both from the experimental and numerical point of view in [29] and [30].

Thus, taking into account the coupled effects generated by the longitudinal and transversal inflexions, the following displacement field has been derived for slender beams under variable bending moment [1]: 
a)

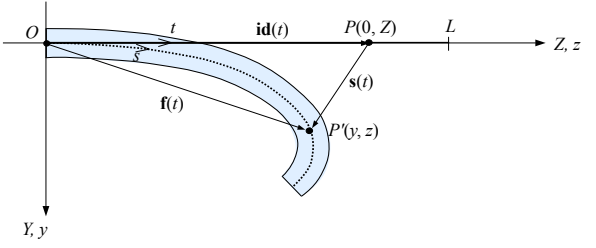

c)

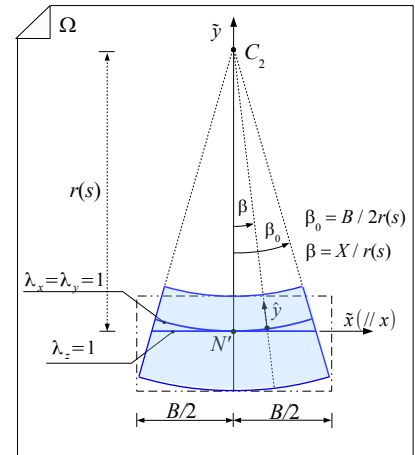

b)
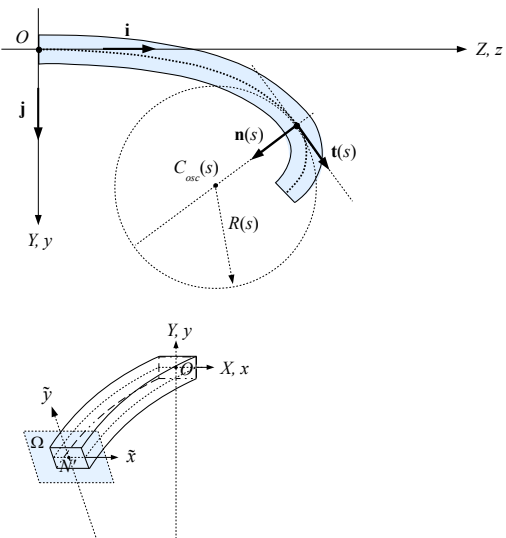

$\theta(s)$

Figure 1: Beam in the deformed configuration. a) Deformation function $\mathbf{f}(t)$ of the beam axis. b) Tangent and normal unit vectors $\mathbf{t}(s)$ and $\mathbf{n}(s)$ along the beam axis. c) Transversal deformation of the generic cross section $\Omega$.

$$
\left\{\begin{array}{l}
u(P)=-X+r(s) e^{-\frac{Y}{r(s)}} \sin \frac{X}{r(s)} \\
v(P)=v(s)-Y+r(s)\left[1-e^{-\frac{Y}{r(s)}} \cos \frac{X}{r(s)}\right] \cos \theta(s) \\
w(P)=w(s)+r(s)\left[1-e^{-\frac{Y}{r(s)}} \cos \frac{X}{r(s)}\right] \sin \theta(s),
\end{array}\right.
$$

for any point $P(X, Y, Z) \in \overline{\mathcal{B}}$. Into system (1), the functions $v(s), w(s)$ and $\theta(s)$, dependent on the curvilinear abscissa $s$ (cf. Fig. 1a), describe displacements and rotations of the beam axis ${ }^{1}$. These functions will be determined separately through system (9). Still in the system (1), $r(s)$ denotes the transversal radius of curvature for the generic cross section (cf. Fig. 1c) and $R(s)$ the longitudinal radius of curvature of the beam axis (cf. Fig. 1b).

Using system (1), a Lagrangian analysis provided the following expressions for stretches and Piola-Kirchhoff stresses [1]: ${ }^{2}$

\footnotetext{
${ }^{1}$ The function $v(s)$ for $v(0,0, Z), w(s)$ for $w(0,0, Z)$ and $\theta(s)$ for $\theta(0,0, Z)$ have been written with abuse of notation. Since the beam axis is inflexed in the vertical plane $X=0$, the component $u(s)$ in the first equation of the system (1) is null.

${ }^{2}$ The following derivatives were used to calculate the displacement gradient:

$$
\frac{\partial v(s)}{\partial s}=-\sin \theta(s), \quad \frac{\partial w(s)}{\partial s}=-1+\cos \theta(s),
$$
}




$$
\begin{aligned}
& \left\{\begin{array}{l}
\lambda_{X}=\lambda_{Y}=e^{-\frac{Y}{r(s)}} \\
\lambda_{Z}=1+\frac{r(s)}{R(s)}\left[1-e^{-\frac{Y}{r(s)}} \cos \frac{X}{r(s)}\right]
\end{array}\right. \\
& {\left[\mathbf{T}_{R}\right]=[\mathbf{R S}]=} \\
& {\left[\begin{array}{ccc}
\cos \frac{X}{r(s)} & -\sin \frac{X}{r(s)} & 0 \\
\sin \frac{X}{r(s)} \cos \theta(s) & \cos \frac{X}{r(s)} \cos \theta(s) & -\sin \theta(s) \\
\sin \frac{X}{r(s)} \sin \theta(s) & \cos \frac{X}{r(s)} \sin \theta(s) & \cos \theta(s)
\end{array}\right]\left[\begin{array}{ccc}
S_{X} & 0 & 0 \\
0 & S_{Y} & 0 \\
0 & 0 & S_{Z}
\end{array}\right],} \\
& \text { with }\left(\lambda_{X}=\lambda_{Y}=\lambda\right) \\
& \begin{array}{c}
S_{X}=S_{Y}=\frac{2}{\lambda}\left\{\left(\lambda^{2}-1\right) a+\left[\left(\lambda^{2}+\lambda_{Z}^{2}\right) \lambda^{2}-2\right] b+\left(\lambda^{4} \lambda_{Z}^{2}-1\right) c\right\}, \\
S_{Z}=\frac{2}{\lambda_{Z}}\left\{\left(\lambda_{Z}^{2}-1\right) a+2\left(\lambda^{2} \lambda_{Z}^{2}-1\right) b+\left(\lambda^{4} \lambda_{Z}^{2}-1\right) c\right\} .
\end{array}
\end{aligned}
$$

The strictly positive quantities $a, b$ and $c$ are the constitutive parameters of a compressible Mooney-Rivlin material. ${ }^{3}$

The corresponding Eulerian analysis gave the following expressions for stretches and Cauchy stresses [1]:

$$
\begin{gathered}
\left\{\begin{array}{l}
\lambda_{x}=\lambda_{y}=1-\frac{\hat{y}}{r(s)} \\
\lambda_{z}=1+\frac{\tilde{y}}{R(s)},
\end{array}\right. \\
{[\mathbf{T}]=\left[\begin{array}{ccc}
\frac{1}{\lambda \lambda_{z}} & 0 & 0 \\
0 & \frac{1}{\lambda \lambda_{z}} & 0 \\
0 & 0 & \frac{1}{\lambda^{2}}
\end{array}\right]\left[\begin{array}{ccc}
S & 0 & 0 \\
0 & S & 0 \\
0 & 0 & S_{z}
\end{array}\right],}
\end{gathered}
$$

where $S$ and $S_{z}$ are given by (4) with stretches in the Eulerian form (5). For the generic cross section, the variables $\hat{y}$ and $\tilde{y}$ are shown in Fig. 1c.

In the set of equations (1)-(6), the anticlastic radius $r(s)$ can be evaluated through the following formula:

$$
\begin{gathered}
r(s)=\frac{a+3 b+2 c}{b+c} R(s), \\
\frac{\partial \sin \theta(s)}{\partial s}=\frac{1}{R(s)} \cos \theta(s), \quad \frac{\partial \cos \theta(s)}{\partial s}=-\frac{1}{R(s)} \sin \theta(s) .
\end{gathered}
$$

In addition, the transversal radius $r(s)$ must not be understood as an independent function in the variable $s$. In fact, in the kinematic model the radius $r(s)$ is evaluated by means of a constitutive relation which exclusively involves the local value of the longitudinal radius $R(s)$ and is not affected by the latter's variations along the beam axis, i.e. $\frac{\partial r(s)}{\partial s}=0$.

${ }^{3}$ A compressible Mooney-Rivlin stored energy function has the following form [31], [32] and [33]:

$$
\omega\left(I_{1}, I_{2}, I_{3}\right)=a I_{1}+b I_{2}+c I_{3}-\frac{d}{2} \ln I_{3}
$$

where $I_{1}=\lambda_{X}^{2}+\lambda_{Y}^{2}+\lambda_{Z}^{2}, I_{2}=\lambda_{X}^{2} \lambda_{Y}^{2}+\lambda_{X}^{2} \lambda_{Z}^{2}+\lambda_{Y}^{2} \lambda_{Z}^{2}, I_{3}=\lambda_{X}^{2} \lambda_{Y}^{2} \lambda_{Z}^{2}$ and $d=2(a+2 b+c)$. This last condition has been determined by imposing that when the stretches are unitary the stresses are null (see, e.g., [34], [35] and [36]). Other details on the above stored energy function can be found in [37] and [38]. 
obtained by imposing the equilibrium conditions [1]. The longitudinal radius $R(s)$ instead remains to be determined.

In [1], integrating the elementary bending moments generated by the principal Cauchy stress $\mathrm{T}_{z}$, orthogonal to the generic cross section in the deformed configuration, a moment-curvature relationship has been obtained. This relationship, derived in the fully nonlinear context of finite elasticity, relates the bending moment $m_{x}(s)$ with the curvature $R(s)^{-1}$, both evaluated along the deformed basic line of the beam. ${ }^{4}$ Although this moment-curvature relationship has a fairly complicated expression, for the class of slender beams it greatly simplifies, assuming a law of direct proportionality between the two nonlinear functions $[1]^{5}$

$$
m_{x}(s)=E_{M R} J_{X} \frac{1}{R(s)},
$$

where $J_{X}$ is the moment of inertia of the cross section with respect to the $X$ axis and $E_{M R}=\frac{4(a+b)(a+4 b+3 c)}{a+3 b+2 c}$. Formula (8) is of crucial importance as it considerably facilitates the nonlinear analysis of high-flexible structures. However, it should be kept in mind that both $m_{x}(s)$ and $R(s)$ depend on the curvilinear abscissa $s$ of the deformed beam axis and that the position of this axis is not known a priori, but it depends on the solution of the equilibrium problem. This causes inevitably couplings and nonlinearities. ${ }^{6}$

The curvature in (8) is determined in geometrically exact manner through the derivative of the rotation $\theta(s)[1]: R(s)^{-1}=\theta^{\prime}(s)=k(s)$. Both displacement components $v(s)$ and $w(s)$ are expressed in terms of rotation $\theta(s)$, which in turn is calculated by means of (8) integrating the curvature $R(s)^{-1}$. The two displacement components $v(s)$ and $w(s)$ allow evaluating the position of the deformed axis of beam, with respect to which the bending moment $m_{x}(s)$ is determined. From the above considerations, the following coupled set of three equations in integral form has been derived [1]:

$$
v(s)=v(0)-\int_{0^{+}}^{s} \sin \theta(\varsigma) d \varsigma,
$$

\footnotetext{
${ }^{4}$ The basic line of the beam is the curve with longitudinal stretch $\lambda_{Z}$ equal to one. Therefore, in the case of inflexed beams, although the beam itself is not, its basic line is inextensible. In the case of slender beams, the basic line coincides with the beam axis, i.e. with the curve that collects the centroids of the cross sections [1].

${ }^{5}$ The applicability limits of relationship (8) are discussed in [1].

${ }^{6} \mathrm{By}$ imposing the conditions of smallness of the displacement and deformation fields and adopting the classic Navier constitutive law, formula (8) transforms into the well-known linear moment-curvature relationship [29]

$$
m_{X}(Z)=E J_{X} \frac{d^{2} v}{d Z^{2}}
$$

where the curvature $\frac{1}{R(Z)}=\frac{d^{2} v}{d Z^{2}}$ is evaluated in an approximate way, neglecting $\frac{d v}{d Z}$ and ignoring the displacement $w(Z)$; the bending moment $m_{X}(Z)$ is evaluated with respect to the undeformed configuration of the beam and the constant $E$ indicates the Young modulus. Since compatibility and equilibrium conditions have been used to obtain this formula, in linear theory, the statically indeterminate structures can be solved using it.
} 


$$
\begin{gathered}
w(s)=w(0)-\int_{0^{+}}^{s}(1-\cos \theta(\varsigma)) d \varsigma, \\
\theta(s)=\int_{0}^{s} k(\varsigma) d \varsigma=\theta(0)+\frac{1}{E_{M R} J_{X}} \int_{0^{+}}^{s} m(\varsigma) d \varsigma .
\end{gathered}
$$

The first two equations are obtained by integrating the following infinitesimal displacements: $d v=-\sin \theta d s, d w=-(1-\cos \theta) d s$, generated by the rotation of an infinitesimal and inextensible horizontal element; while the third equation derives from the integration of (8).

Once system (9) has been recalled, the equilibrium problem of high-flexible beams can be handled as follows. The first step concerns the resolution of system (9) through the iterative numerical procedure illustrated in Appendix A of [1]. This initial computation provides the displacements and rotations of the beam axis, $v(s), w(s)$ and $\theta(s)$, and the bending moment distribution along this deformed axis, $m_{x}(s)$, which can be evaluated according to the specific case treated. Once the moment $m_{x}(s)$ is known, as mentioned above, also the radii $R(s)$ and $r(s)$ can be calculated through (8) and (7). The second step deals with the determination of the three-dimensional shape assumed by the beam in the deformed configuration. Since functions $v(s), w(s), \theta(s), R(s)$ and $r(s)$ are available, the displacements of each point of the beam can be evaluated with (1). The third step is dedicated to the analysis of stretches and stresses for each point of the cross sections. For this purpose, the Lagrangian expressions (2) and (3) or the Eulerian expressions (5) and (6) can be used.

Note that in the integral form, system (9) requires a minor degree of regularity of the functions involved with respect to a corresponding differential formulation. For example, with (9) it is possible to consider structures in which the distributed external load presents jump discontinuities.

\section{External loads ${ }^{7}$}

The deformation of the beam axis can be defined by the deformation function f : $\mathscr{L} \rightarrow \mathscr{L}^{\prime}$, such that

$$
\mathbf{f}(t)=(y(t), z(t)),
$$

with $y(t)=v(t)$ and $z(t)=t+w(t), t \in \mathscr{L}$ (cf. Fig. 1a). The parametric curve $\mathbf{f}(t)$, which describes the shape of the deformed beam axis, is supposed to be smooth $\left(\mathbf{f} \in C^{1}\right)$ and injective. Each smooth curve can be re-parameterized with respect to the arc length $s(t): \mathbf{f}(t(s))=\mathbf{f}(s)$. Among the different ways of expressing the curve, the parametrized form with respect to the arc length $\mathbf{f}(s)$

\footnotetext{
${ }^{7}$ In this Section the following notation is introduced: $(\cdot)^{\prime}$, the apex indicates that the function $(\cdot)$ is defined in the deformed configuration; $(\cdot)^{(1)}$, the superscript denotes the derivative with respect to the curvilinear abscissa $s,(\cdot)^{(1)}=\frac{d(\cdot)}{d s}$, and $(\cdot)^{(2)}$ the second derivative, $(\cdot)^{(2)}=\frac{d^{2}(\cdot)}{d s^{2}}$.
} 
a)

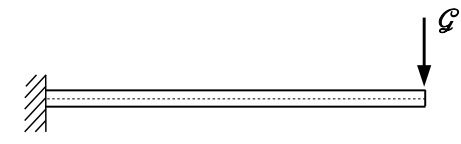

b)

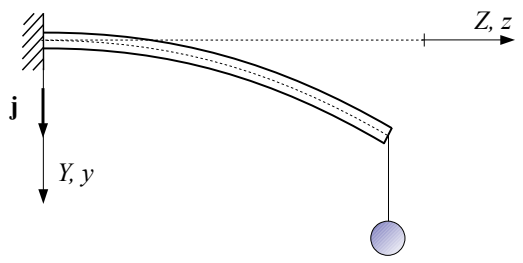

Figure 2: Cantilever beam subjected to the concentrated dead load $\mathcal{G}$. a) Reference configuration. b) Deformed configuration.

is the more suitable to study the geometrical properties of the curve itself [1]. The derivative of $\mathbf{f}(s)$ is known as tangent vector $\mathbf{t}(s)=\mathbf{f}^{(1)}(s)$ (cf. Fig. 1b). Since the beam axis is inextensible, $\left\|\mathbf{f}^{(1)}(s)\right\|=1$, that is, for any $s$, the tangent vector $\mathbf{t}(s)$ has unitary modulus. Having $\mathbf{t}(s)$ constant modulus, its derivative $\mathbf{t}^{(1)}(s)=\mathbf{f}^{(2)}(s)$ is orthogonal to $\mathbf{t}(s)$ and generally has no unitary modulus. The normal unit vector $\mathbf{n}(s)$ can thus be defined as: $\mathbf{n}(s)=\mathbf{f}^{(2)}(s) /\left\|\mathbf{f}^{(2)}(s)\right\|$ (cf. Fig. 1b). The two unit vectors $\mathbf{t}(s)$ and $\mathbf{n}(s)$, defined in the deformed configuration, are useful in assigning live loads.

Although there is no a clear definition in the Literature, a dead load is generally distinguished from a live one because it does not depend on the deformation. To fix the ideas, let us consider the simple case of a cantilever beam with a constant concentrated force $\mathcal{G}$ applied at its free end (cf. Fig. 2a). To define a dead load, it can be imagined that $\mathcal{G}$ models a hanging mass subjected to gravity. This weight force moves parallel to itself during deformation, preserving modulus and direction (cf. Fig. 2b). Its application point in the deformed configuration takes the following coordinates: $(L+w(L), v(L))$, and is known only when the equilibrium problem is solved.

Likewise, a distributed load is a dead load if every elementary force $\mathbf{g}(t) d t$ preserves modulus and direction during the deformation. The vectorial function g: $\mathscr{L} \rightarrow \mathcal{V}$ (where $\mathscr{L} \equiv[0, L]$ is the undeformed beam axis and $\mathcal{V}$ the vector space) denotes the density of the distributed load per unit length of the beam in the reference configuration. Obviously, even the elementary forces $\mathbf{g}(t) d t$ can move parallel to themselves. The corresponding density $\mathbf{g}^{\prime}(s, \mathbf{f}(s))$, with $s \in \mathscr{L}^{\prime} \equiv[0, L]$ and $\mathbf{f} \in \mathcal{V}$, in the deformed configuration depends also on the deformation function $\mathbf{f}(s)$ and it can be expressed locally through the following relationship:

$$
\mathbf{g}(t) d t=\mathbf{g}^{\prime}(s, \mathbf{f}(s)) d s,
$$

which translates the invariance of elementary forces. In particular, in our situation, being the beam axis inextensible, i.e. $d t=d s$, also the density does not 
change with respect to the deformed configuration: $\mathrm{g}=\mathrm{g}^{\prime}$.

The assumption that the applied forces are dead loads is a simplification from the mathematical standpoint, since they are known functions in the reference configuration. However, it should be kept in mind that actual applied forces can seldom be modeled as dead loads, because they also depend on the deformation itself. In other words, the variety of live loads is much wider than that of dead loads.

Unlike a dead load, assigning a live load does not mean specifying the entity of an external action, but rather prescribing a law of variation of the action as a function of the deformation for every conceivable configuration.

For the simple case of Fig. 2, it can be requested, for example, that the concentrated force $\mathcal{G}$, with constant modulus $\mathcal{G}=\|\mathcal{G}\|$, is always orthogonal to the deformed axis of the beam: $\mathcal{G}=\mathcal{G} \mathbf{n}(L)$. The case in which the force remains parallel to the deformed beam axis: $\mathcal{G}=\mathcal{G} \mathbf{t}(L)$, or that it retains a certain inclination: $\mathcal{G}=\mathcal{G}(\sin \gamma) \mathbf{n}(L)+\mathcal{G}(\cos \gamma) \mathbf{t}(L)$ (where $\gamma$ is the angle between the direction of the force and the tangent to the deformed beam axis), can be treated similarly. In any case, the effective direction assumed by the force will be known with the resolution of the equilibrium problem.

A distributed live load is characterized by a density per unit length of the beam in the deformed configuration $\mathrm{g}^{\prime}: \mathscr{L}^{\prime} \times \mathcal{V} \times \mathcal{U} \times \mathcal{U} \rightarrow \mathcal{V}$ (where $\mathcal{U}$ is the space of unit vectors), which in general depends on the curvilinear abscissa $s$, on the deformation function $\mathbf{f}(s)$ and on its derivatives $\mathbf{f}^{(1)}(s)$ and $\mathbf{f}^{(2)}(s)$. By way of example, for a uniformly distributed load, $\left\|\mathbf{g}^{\prime}\right\|=g^{\prime}=$ const., the following cases can be considered: $\mathbf{g}^{\prime}\left(s, \mathbf{f}(s), \mathbf{f}^{(2)}(s)\right)=g^{\prime} \mathbf{n}(s)$, that is, a load always orthogonal to the deformed beam axis (like a hydrostatic pressure of a fluid); $\mathbf{g}^{\prime}\left(s, \mathbf{f}(s), \mathbf{f}^{(1)}(s)\right)=g^{\prime} \mathbf{t}(s)$, that is, a load always parallel to the deformed beam axis (like a viscous effect of a fluid); $\mathbf{g}^{\prime}\left(s, \mathbf{f}(s), \mathbf{f}^{(1)}(s), \mathbf{f}^{(2)}(s)\right)=g^{\prime}(\sin \gamma) \mathbf{n}(s)+$ $g^{\prime}(\cos \gamma) \mathbf{t}(s)$, that is, a load that changes its direction keeping the inclination fixed with respect to the deformed beam axis (like the combination of the two previous effects). ${ }^{8}$ The few examples above discussed obviously do not exhaust the casuistry, which, as can be imagined, is very wide, being it possible to assign in many ways the laws of variation of intensity and direction of these applied forces.

Finally, it is observed that in the case of dead loads, the force balance does not change passing from the deformed configuration to the reference one, while for live loads it always varies. The moment balance varies both with dead and live loads.

In the next Sections, dead and live loads will be applied to high-flexible structures, distinguishing between isogeometric and hypergeometric beams.

\footnotetext{
${ }^{8}$ If the rotation $\theta(s)$ is added to the angle $\gamma$ then the load retains its direction. In this case, the load can simulate for example the action of a stationary wind.
} 


\section{Isogeometric beams}

In linear theory, as is well known, the static equilibrium equations are sufficient to solve statically determinate structures. From this circumstance the name of this class of structures derives. This is not true in nonlinear theory, since equilibrium equations are no longer sufficient. In fact, in addition to equilibrium equations, compatibility conditions must also be satisfied even in the case of statically determinate structures, being necessary integrating the moment-curvature relationship (8) in any case. Consequently, the classical classification between statically determinate and indeterminate structures of linear theory loses its meaning when beams in nonlinear theory are considered. This essentially because for both classes of structures it is necessary to impose compatibility and equilibrium conditions together. Therefore, contrary to what is generally done in linear theory, a classification between isogeometric and hypergeometric structures appears more coherent. In the case of the isogeometric structures, the degree of kinematic constraints is strictly necessary to prevent any rigid displacement when the beams of the structure are imagined like rigid bodies. When this number is exceeded, the structure is hypergeometric.

For a single beam span, the internal actions (i.e. normal force $n$, shear force $t$ and bending moment $m$ ) at the generic curvilinear abscissa $s$ in the deformed configuration (cf. Fig. 1a) can be evaluated with the following formulae:

$$
\begin{gathered}
n(s)=n(0) \cos [\theta(s)-\theta(0)]+t(0) \sin [\theta(s)-\theta(0)] \\
-\int_{0^{+}}^{s}\left[\mathbf{g}^{\prime}(\varsigma) \cdot \mathbf{i} \cos \theta(s)+\mathbf{g}^{\prime}(\varsigma) \cdot \mathbf{j} \sin \theta(s)\right] d \varsigma \\
t(s)=-n(0) \sin [\theta(s)-\theta(0)]+t(0) \cos [\theta(s)-\theta(0)] \\
\quad+\int_{0^{+}}^{s}\left[\mathbf{g}^{\prime}(\varsigma) \cdot \mathbf{i} \sin \theta(s)-\mathbf{g}^{\prime}(\varsigma) \cdot \mathbf{j} \cos \theta(s)\right] d \varsigma \\
m(s)=m(0)-[n(0) \cos \theta(0)-t(0) \sin \theta(0)][v(s)-v(0)] \\
+[n(0) \sin \theta(0)+t(0) \cos \theta(0)][s+w(s)-w(0)] \\
+\int_{0^{+}}^{s}\left\{\mathbf{g}^{\prime}(\varsigma) \cdot \mathbf{i}[v(s)-v(\varsigma)]-\mathbf{g}^{\prime}(\varsigma) \cdot \mathbf{j}[s-\varsigma+w(s)-w(\varsigma)]\right\} d \varsigma
\end{gathered}
$$

From the first two equations it can be noted how the normal and shear forces are always coupled. Both the normal and shear force contribute to the determination of the bending moment. These considerations are consequences of the fact that internal actions are evaluated with respect to the deformed beam axis which is curvilinear. 
Introducing $(12)_{3}$ into $(9)_{3}$, the set of equations to compute the position of the deformed beam axis for isogeometric beams is obtained ${ }^{9}$. As above mentioned, because this system in the three unknown functions $v(s), w(s)$ and $\theta(s)$ is coupled and highly nonlinear, it is solved with the recursive numerical procedure illustrated in [1]. Once the position of the deformed axis (i.e. the so-called deflection curve) has been determined, the first step of the procedure illustrated at the end of Section 2 was performed. Given the (12), the internal actions in the deformed configuration are known. Then, following the procedure, the shape assumed by the beam in the deformed configuration, the stretches and the stresses can be computed.

In the following some applications are performed considering two typical structural systems: The cantilever beam and the simply supported beam. Both structures will be subjected to uniformly distributed dead and live loads. The live loads will be such as to always remain orthogonal to the deformed axis of the beam. The following geometrical and constitutive parameters are adopted for the beam: $B=2, H=1, L=15$ and $a=1, b=0.05, c=2.256$. These constitutive parameters are related to a specific rubber-like material studied in [29].

The cantilever beam under the dead loads is shown in Fig. 3. Using (9) and (12), with

$$
v(0)=w(0)=\theta(0)=0, \quad \mathbf{g}^{\prime}(s)=g^{\prime}(s) \mathbf{j},
$$

the deflection curves and the internal actions have been computed. ${ }^{10}$ In the reference configuration, the modulus of the load density is constant: $g(t)=\mu q$, where $\mu$ is the load multiplier. Fig. 3a shows the profiles of the beam, in the vertical plane $x=0$, as $\mu$ increases. The intensity of the color grows by increasing the value of $\mu$. The graphical representations of each deformed beam, including its thickness, are drawn exactly in scale. The first case, computed for $\mu=1$, has been obtained by imposing a vertical displacement of the free end, $v(L)$, equal to $1 / 100$ of the length of the beam. Note that, although the displacements are not small, the results for this first value of $\mu$ are almost similar to those given by the linear theory: The diagram of normal force, of shear force and of bending moment are null, linear and quadratic, respectively. As $\mu$ increases, the displacements grow and the deformed beam moves away more and more from the undeformed configuration. For high values of $\mu$, the terminal part of the beam tends to assume an almost vertical position and the convergence of the numerical method becomes very slow. The values displayed in the graphs of Fig. 3b, 3c, 3d and 3e refer to the curved axis of the deformed beam and, for reasons of clarity of the graphical representation, they have been reported on a horizontal axis. In Fig. 3b, for each value of $\mu$, the intensity of the load density

\footnotetext{
${ }^{9}$ In a slender beam the deformation effects generated by the bending moment are more important than those produced by normal force and shear force, which, for this reason, are generally neglected.

${ }^{10}$ This first step of the analysis, aimed at the determination of deflections curves, can also be performed using a nonlinear Elastica equation, i.e. evaluating the curvature in a geometrically exact manner and calculating the bending moment with respect to the deformed axis. This is as long as the curvatures are sufficiently small[1].
} 
along the deformed beam axis, $\bar{g}^{\prime}(s)$, re-evaluated for a horizontal infinitesimal element on the basis of the following relationship: $\bar{g}^{\prime} \cos \theta d s=g^{\prime} d s$, with $\cos \theta=\mathbf{i} \cdot \mathbf{t}$, is indicated. These values grow at the free end of the beam where the rotations are greater. Fig. 3c illustrates the diagrams of normal force as $\mu$ varies. Note that, in nonlinear theory, the normal force is not null along the beam axis. The diagrams are null at the ends and have the maximum in an intermediate position. Fig. 3d and 3e show the diagrams of the shear force and bending moment. In both diagrams the maximum values occur at the clamped end. The Fig. 3f and $3 \mathrm{~g}$ are related to the cross section subjected to the maximum bending moment, which occurs at $z=0$. In Fig. 3f, the vertical diagram of $\left(\lambda_{z}(0, y, 0)-1\right)$ and the line with $\lambda_{z}=1$ (which characterizes the points with no longitudinal deformation - neutral axis for the deformation) are reported. Both these lines are rectilinear according to the hypothesis of conservation of the planarity of the cross sections. On the basis of this hypothesis, in fact, the cross sections rotate rigidly (with a large angle) around the neutral axis, exhibiting an anticlastic flexion in their own plane. The values of the stretches at the ends of the vertical segment passing through the centroid are indicated. The longitudinal Cauchy stress $\mathrm{T}_{z}$ is shown in Fig. $3 \mathrm{~g}$ for each point of the cross section by means of contour lines. The stress component $\mathrm{T}_{z}$ assumes the maximum tensile value at the upper edge and that of compression at the lower edge. The neutral line for the stress $\left(\mathrm{T}_{z}=0\right)$ does not coincide with the neutral axis for the deformation $\left(\lambda_{z}=1\right)$.

Let's consider the same cantilever beam but subjected now to live loads. Using (9) and (12), with

$$
v(0)=w(0)=\theta(0)=0, \quad \mathbf{g}^{\prime}(s)=g^{\prime}(s) \mathbf{n}(\mathrm{s}),
$$

the deflection curves and the internal actions have been computed and reported in Fig. 4. In the deformed configuration, the intensity of the load density is constant: $g^{\prime}(s)=\mu q$. Note from Fig. 4a how the deflections curves tend, with respect to the previous case, to curl themselves as $\mu$ grows. In Fig. $4 \mathrm{~b}$, the vertical and horizontal components of the load density, $g_{h}^{\prime}=g^{\prime} \sin \theta$ and $g_{v}^{\prime}=g^{\prime} \cos \theta$, are displayed. As $\mu$ increases, the vertical component changes sign along the beam axis. In fact, in the terminal part of the beam, the load makes an almost complete rotation. As can be seen from Fig. 4c, the normal force is now compressive and is not zero at the clamped end. The shear force changes sign along the beam axis (see Fig. 4d) and the maximum of the diagram, as $\mu$ grows, is in an intermediate position. The bending moment diagrams are shown in Fig. 4e and again, for high $\mu$ values, the maximum moves to an intermediate position $^{11}$. For this second application with live loads the convergence of the numerical process becomes slower.

\footnotetext{
${ }^{11}$ For this case, the correctness of the normal and shear force at the clamped end can be checked later. In fact, taking a deflection curve, a straight horizontal segment and a vertical ones can be traced joining the free end of the beam with the clamped section, in order to realize a closed simply connected profile. Now we can think that this profile is subjected to a uniform pressure $g^{\prime}$, therefore the normal and shear force at the clamped end are the resultants of the pressures on the vertical and horizontal segments, respectively.
} 

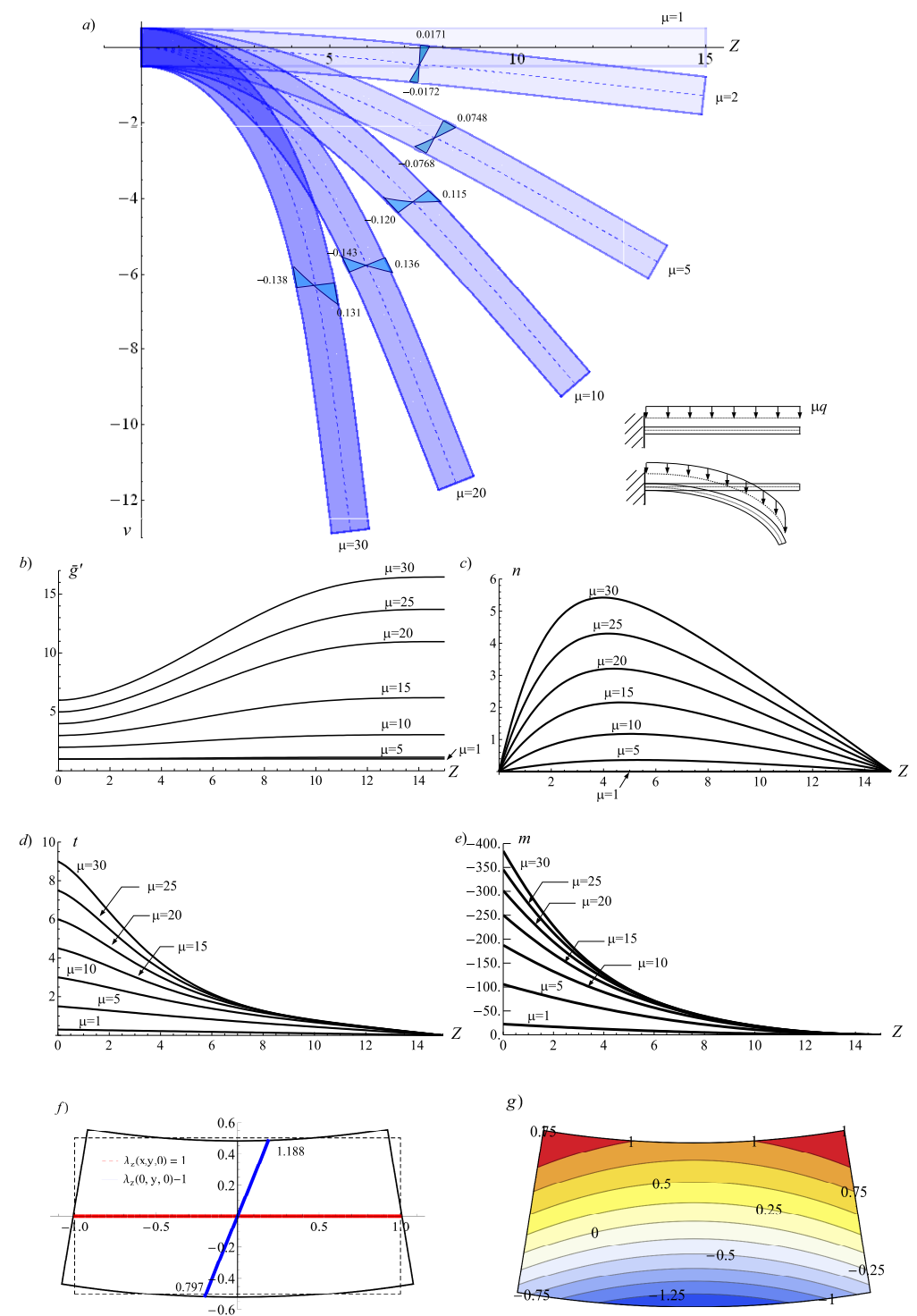

Figure 3: Cantilever beam under uniformly distributed vertical load with variable load multiplier $\mu$. Case with dead loads. a) Deflection curves. b) Intensity of the load density $\bar{g}^{\prime}$ along the deformed beam axis. c) Normal force diagrams. d) Shear force diagrams. e) Bending moment diagrams. f) Eulerian stretches for the cross section with maximum bending moment. Vertical diagram of $\left(\lambda_{z}(0, y, 0)-1\right)$ and neutral axis for the deformation. $\left.g\right)$ Cauchy stress component $\mathrm{T}_{z}$ for the cross section with maximum bending moment. 

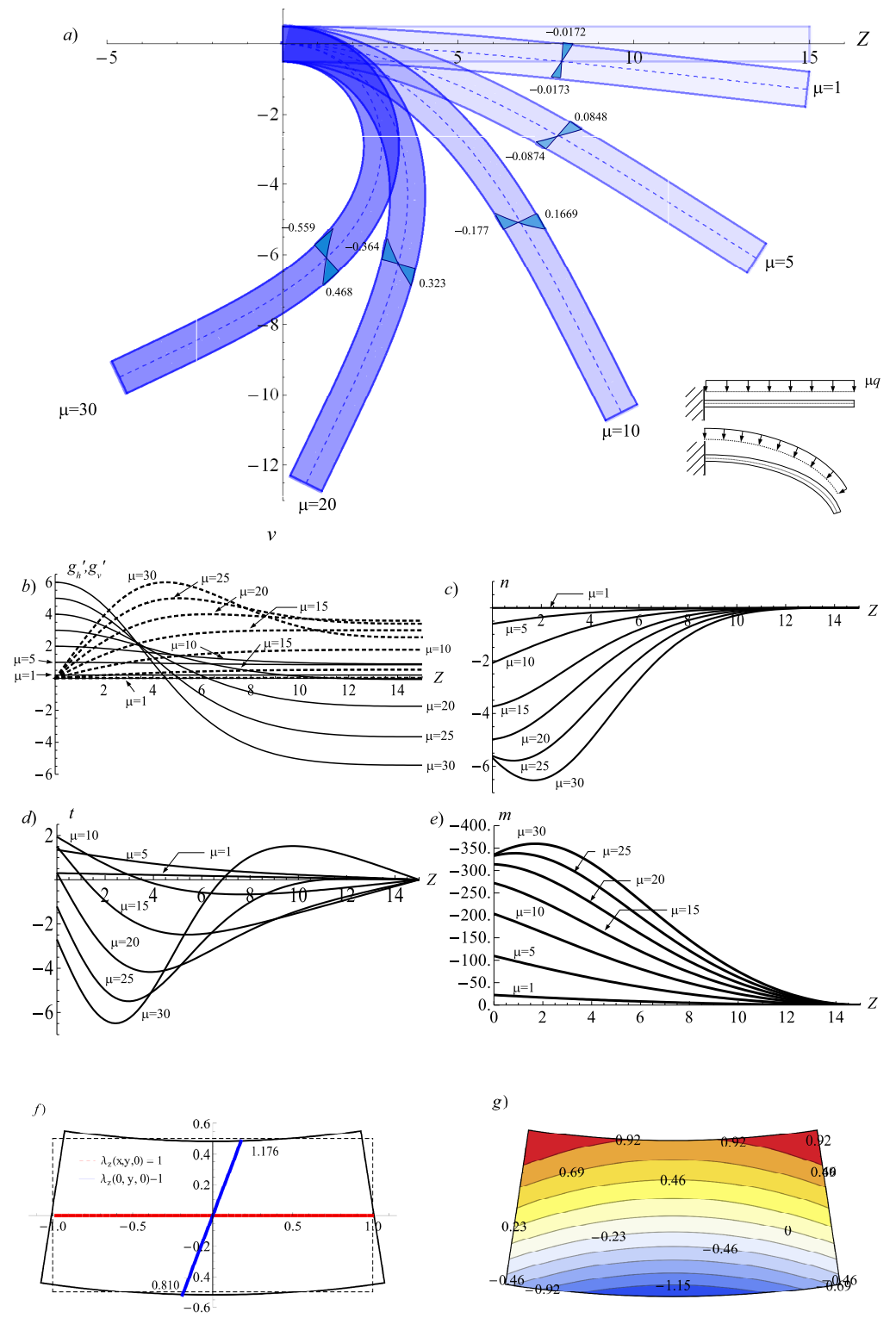

Figure 4: Cantilever beam under uniformly distributed load with variable load multiplier $\mu$. Case with live loads. The loads act orthogonally to the deformed beam axis. a) Deflection curves. b) Intensity of the load density along the deformed beam axis. The vertical components are represented by the family of continuous lines, the horizontal components by the dotted lines. c) Normal force diagrams. d) Shear force diagrams. e) Bending moment diagrams. f) Eulerian stretches for the cross section with maximum bending moment. Vertical diagram of $\left(\lambda_{z}(0, y, 0)-1\right)$ and neutral axis for the deformation. $\left.g\right)$ Cauchy stress component $\mathrm{T}_{z}$ for the cross section with maximum bending moment. 

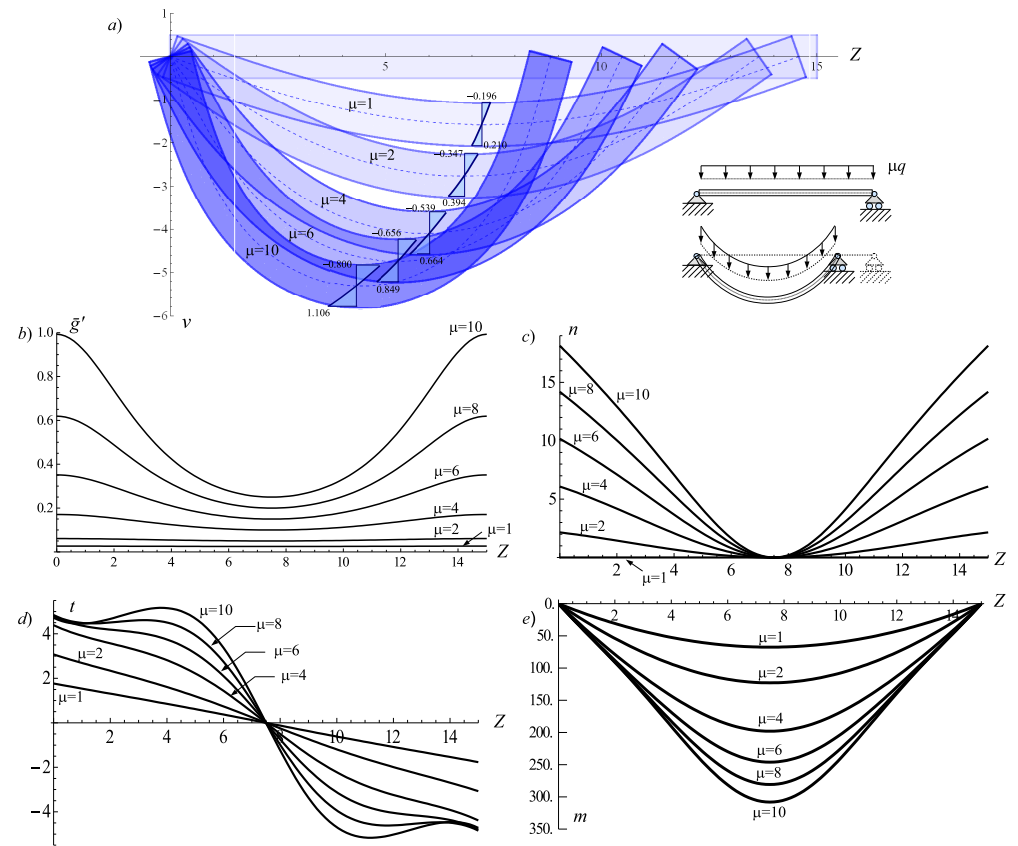

f)
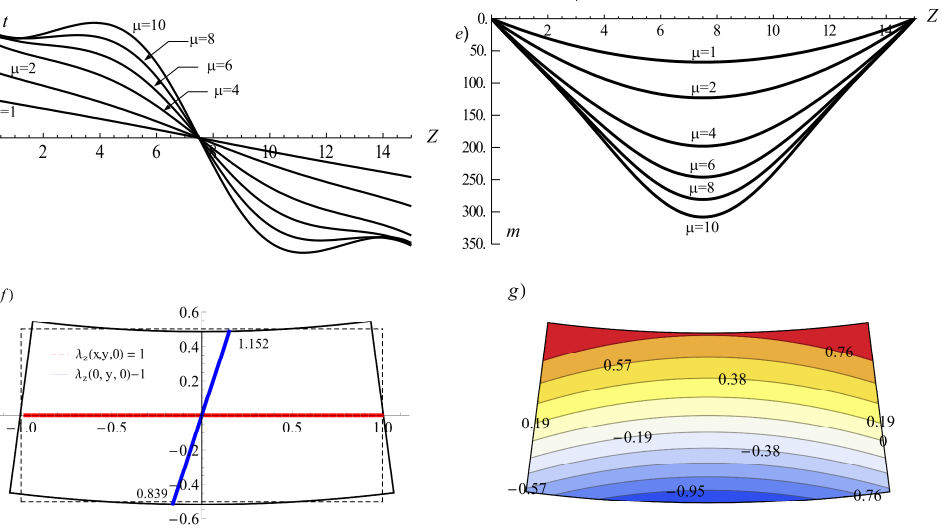

g)

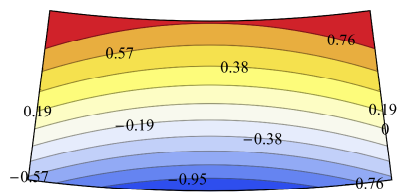

Figure 5: Simply supported beam under uniformly distributed vertical load with variable load multiplier $\mu$. Case with dead loads. a) Deflection curves. $b$ ) Intensity of the load density $\bar{g}^{\prime}$ along the deformed beam axis. c) Normal force diagrams. d) Shear force diagrams. e) Bending moment diagrams. f) Eulerian stretches for the cross section with maximum bending moment. Vertical diagram of $\left(\lambda_{z}(0, y, 0)-1\right)$ and neutral axis for the deformation. $\left.g\right)$ Cauchy stress component $\mathrm{T}_{z}$ for the cross section with maximum bending moment. 

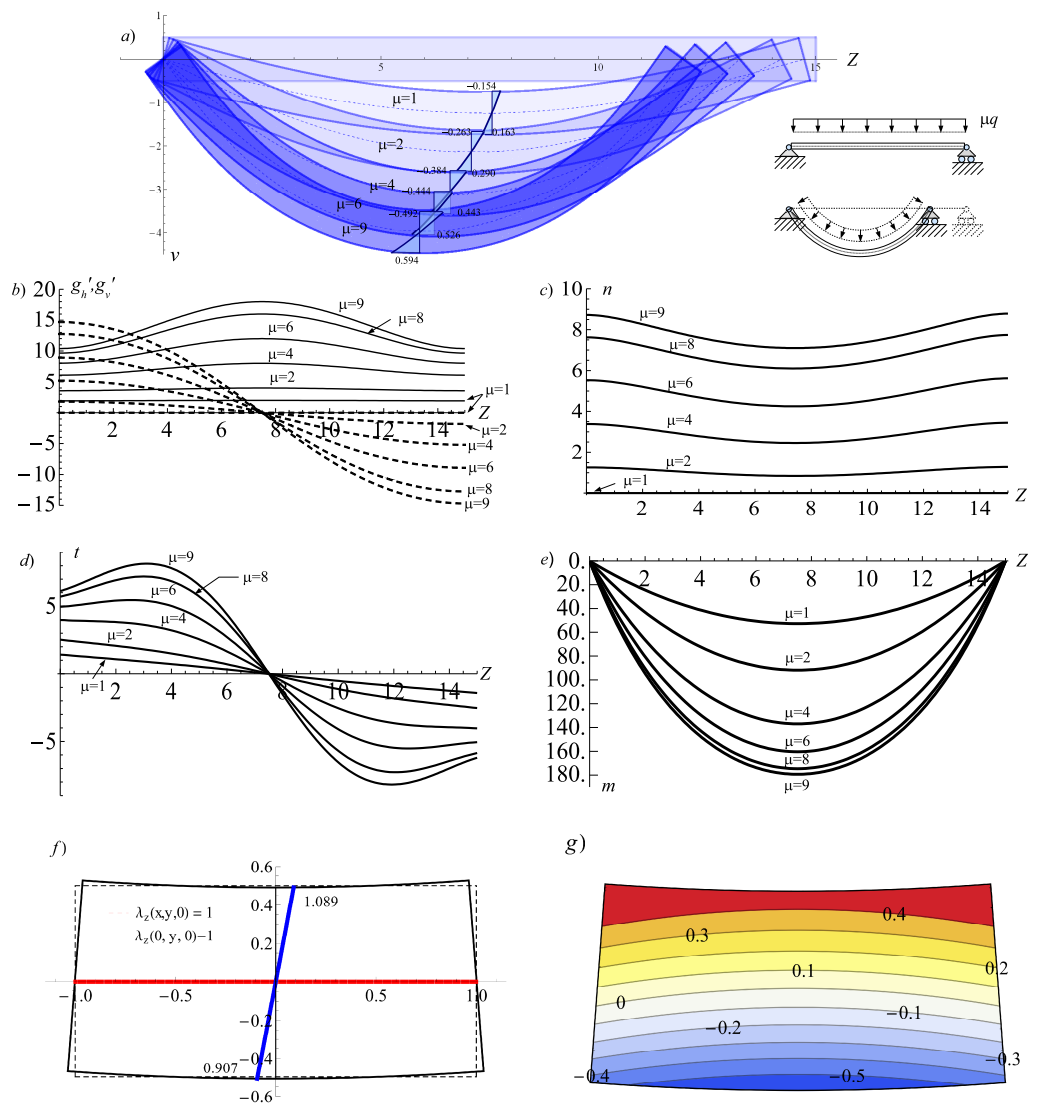

g)

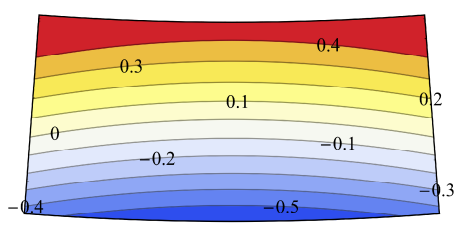

Figure 6: Simply supported beam under uniformly distributed load with variable load multiplier $\mu$. Case with live loads. The loads act orthogonally to the deformed beam axis. a) Deflection curves. $b$ ) Intensity of the load density along the deformed beam axis. The vertical components are represented by the family of continuous lines, the horizontal components by the dotted lines. c) Normal force diagrams. d) Shear force diagrams. e) Bending moment diagrams. f) Eulerian stretches for the cross section with maximum bending moment. Vertical diagram of $\left(\lambda_{z}(0, y, 0)-1\right)$ and neutral axis for the deformation. $\left.g\right)$ Cauchy stress component $\mathrm{T}_{z}$ for the cross section with maximum bending moment. 
Fig. 5 illustrates the case of a simply supported beam subjected to dead loads. For this case

$$
v(0)=w(0)=v(L)=0, \quad \mathbf{g}^{\prime}(s)=g^{\prime}(s) \mathbf{j} .
$$

In addition, the rotation at the middle of the deflection curves, $\theta(L / 2)$, is zero and the modulus of the load density in the reference configuration is constant: $g(t)=\mu q$. Fig. 5a shows the deflection curves. ${ }^{12}$ With the growth of $\mu$, the displacements increase and the right sliding support moves more and more to the left towards the hinge. The first curve, computed for $\mu=1$, has been obtained by imposing a vertical displacement in the middle of the deformed beam, $v(L / 2)$, equal to $1 / 10$ of the length of the beam. The results for this first value of $\mu$ are almost similar to those provided by the linear theory. In Fig. 12b, for each value of $\mu$, the intensity of the load density $\bar{g}^{\prime}(s)$ is indicated. These values grow at the ends of the beam where the rotations are greater. Fig. $5 \mathrm{c}$ shows that along the beam axis there is tensile force. The normal force is maximum at the ends and zero at the middle. As shown in Fig. 5d, the shear force changes the sign along the beam axis and, with the increase of $\mu$, the maximums move from the end sections to more central positions. As $\mu$ increases, the bending moment diagram takes an increasingly acute shape, with the maximum in the middle (see Fig. 5e).

Let's consider the same simply supported beam but now subjected to live loads as shown in Fig. 6. For this case

$$
v(0)=w(0)=v(L)=0, \quad \mathbf{g}^{\prime}(s)=g^{\prime}(s) \mathbf{n}(\mathrm{s}) .
$$

The rotation at the middle of the deflection curves, $\theta(L / 2)$, is zero again and, in the deformed configuration, the intensity of the load density is constant: $g^{\prime}(s)=\mu q$. In Fig. 6b, the vertical and horizontal components of the load density, $g_{h}^{\prime}$ and $g_{v}^{\prime}$, are displayed. The horizontal component changes sign along the axis, assuming the value zero in the middle where the vertical component is instead maximum. The normal force is tensile and, compared to the above case with dead loads, presents a variation along the axis more moderate, without ever becoming null (see Fig. 6c). The shear diagrams are similar to those of the previous case (see Fig. 6d), while the bending moment diagrams have a more rounded shape (see Fig. 6e). ${ }^{13,14}$ Finally, on this example it should be noted that as $\mu$ increases, the external load, especially in the terminal portions of the beam, tends to become increasingly horizontal. But, the horizontal components of the load balance each other, so that, with the growth of $\mu$, the deflection curves tend to approach each other, the normal force grows (but it does not

\footnotetext{
${ }^{12}$ If the bending stiffness tends to zero, the deflection curves approach the shape of the catenary.

${ }^{13}$ If the two ends of a deflection curve are joined with a horizontal segment, a closed profile is obtained. The pressure multiplied by the length of the segment is equal to the sum of the two vertical reactions.

${ }^{14}$ If the flexural stiffness tends to zero, the deflection curves approach the shape assumed by half of the cross section of a thin balloon subjected to internal pressure.
} 
produce deformation, being the beam axis inextensible), the shear force and the bending moment undergo instead small increments.

In the examples discussed above, the law of the bending moment has a unique expression for the entire domain $[0, L]$. However, there are situations in which constraints and concentrated actions are applied along the beam axis. Then, different expressions of the bending moment hold for the single subdomains which make up the beam axis. In these cases it is necessary to impose the (9) for each subdomain and then write the continuity conditions at the interface of each pair of adjacent subdomains: $\Delta w=\Delta v=\Delta \theta=0$.

\section{$5 \quad$ Hypergeometric beams}

To solve a hypergeometric beam it is necessary, initially, to transform it into an isogeometric beam, replacing the redundant constraints with their unknown reactions. The expression of the bending moment will now also depend on the redundant reactions. This expression is then introduced into fundamental system (9) to which compatibility equations at the suppressed redundant constraints must be added. Compatibility equations are equal in number to redundant reactions to be determined. It is important to keep in mind that, unlike linear theory, compatibility equations at the redundant constraints must be imposed in the deformed configuration.

As an example of hypergeometric structure, a beam clamped at one end and supported at the other is considered. This structure has a redundant reaction. Using (9) and (12), with

$$
v(0)=w(0)=\theta(0)=v(L)=0, \quad \mathbf{g}^{\prime}(s)=g^{\prime}(s) \mathbf{j}=\mu q \mathbf{j},
$$

the deflection curves and the internal actions have been computed and reported in Fig. 7. Compared to the previous cases of isogeometric beams, in the compatibility conditions (17) an equation has been added that is used for the calculation of the redundant reaction. Fig. 7a shows the deflection curves. With the growth of $\mu$, the displacements increase and the right sliding support moves more and more to the left. However, the redundant constraint globally reduces the deformability of the beam, which undergoes displacements that are smaller than the previous cantilever beams. In Fig. 7b, the intensity of the load density $\bar{g}^{\prime}(s)$ is indicated. These values grow with increasing rotations. Fig. 7c shows that along the beam axis there is tensile force. The normal force vanishes at the clamped end and in an internal position. Both the shear and bending moment diagrams change sign along the beam axis and assume the maximum values at the clamped end (cf. Fig. 7d and 7e).

The above hypergeometric structure is now solved for the case of live loads: $\mathbf{g}^{\prime}(s)=g^{\prime}(s) \mathbf{n}(\mathrm{s})=\mu q \mathbf{n}(\mathrm{s})$, and the results are shown in Fig. 8. As can be observed from Fig. 8a, the vertical displacements of the curved deflections, compared to the case of dead loads, are smaller. This is due to the fact that the live loads abound the vertical direction and rotate with the beam axis. Since the end portions of the beam rotate in opposite directions, the horizontal 

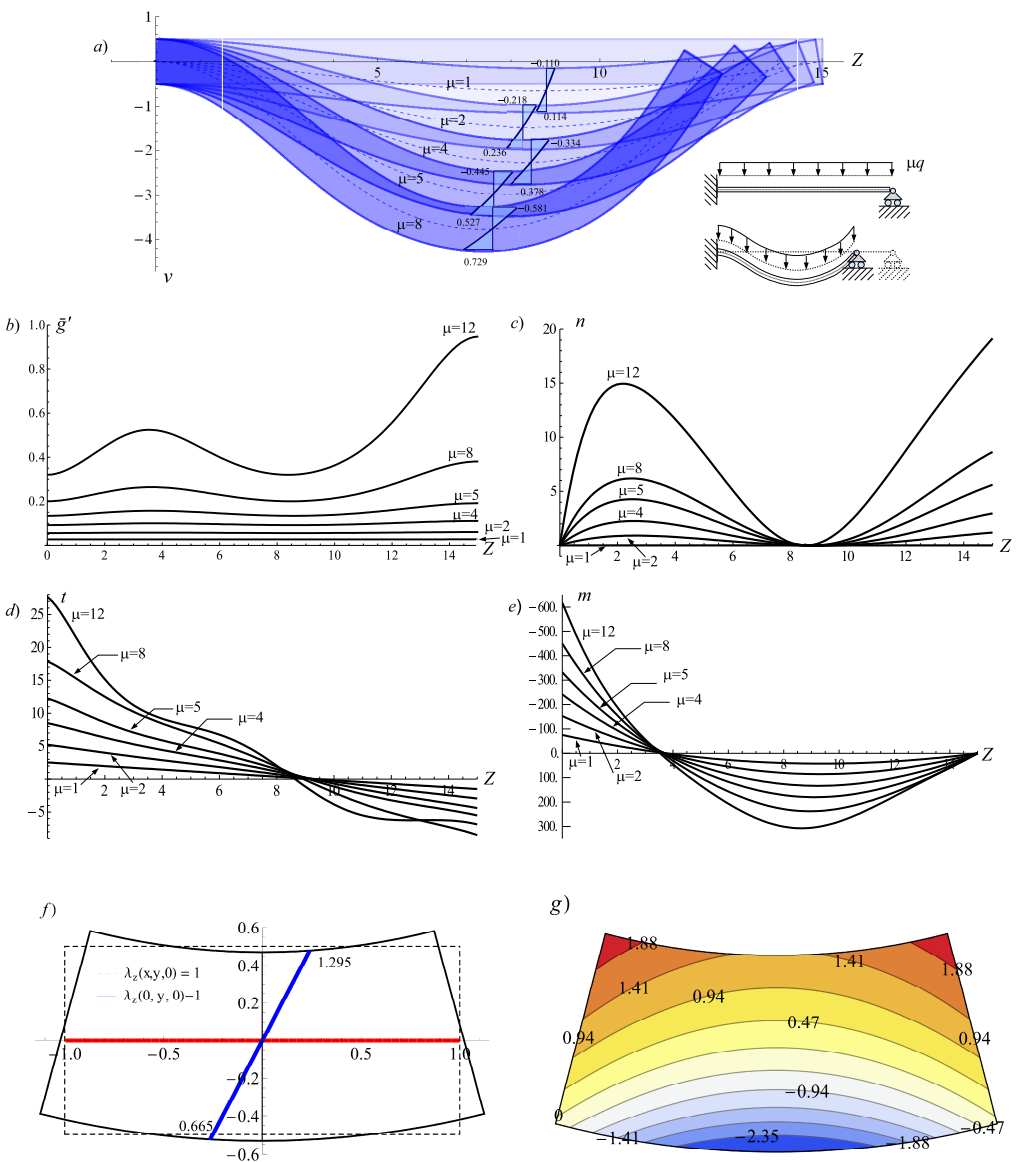

Figure 7: Beam clamped at one end and supported at the other under uniformly distributed vertical load with variable load multiplier $\mu$. Case with dead loads. a) Deflection curves. b) Intensity of the load density $\bar{g}^{\prime}$ along the deformed beam axis. c) Normal force diagrams. d) Shear force diagrams. e) Bending moment diagrams. $f$ ) Eulerian stretches for the cross section with maximum bending moment. Vertical diagram of $\left(\lambda_{z}(0, y, 0)-1\right)$ and neutral axis for the deformation. $g$ ) Cauchy stress component $\mathrm{T}_{z}$ for the cross section with maximum bending moment. 

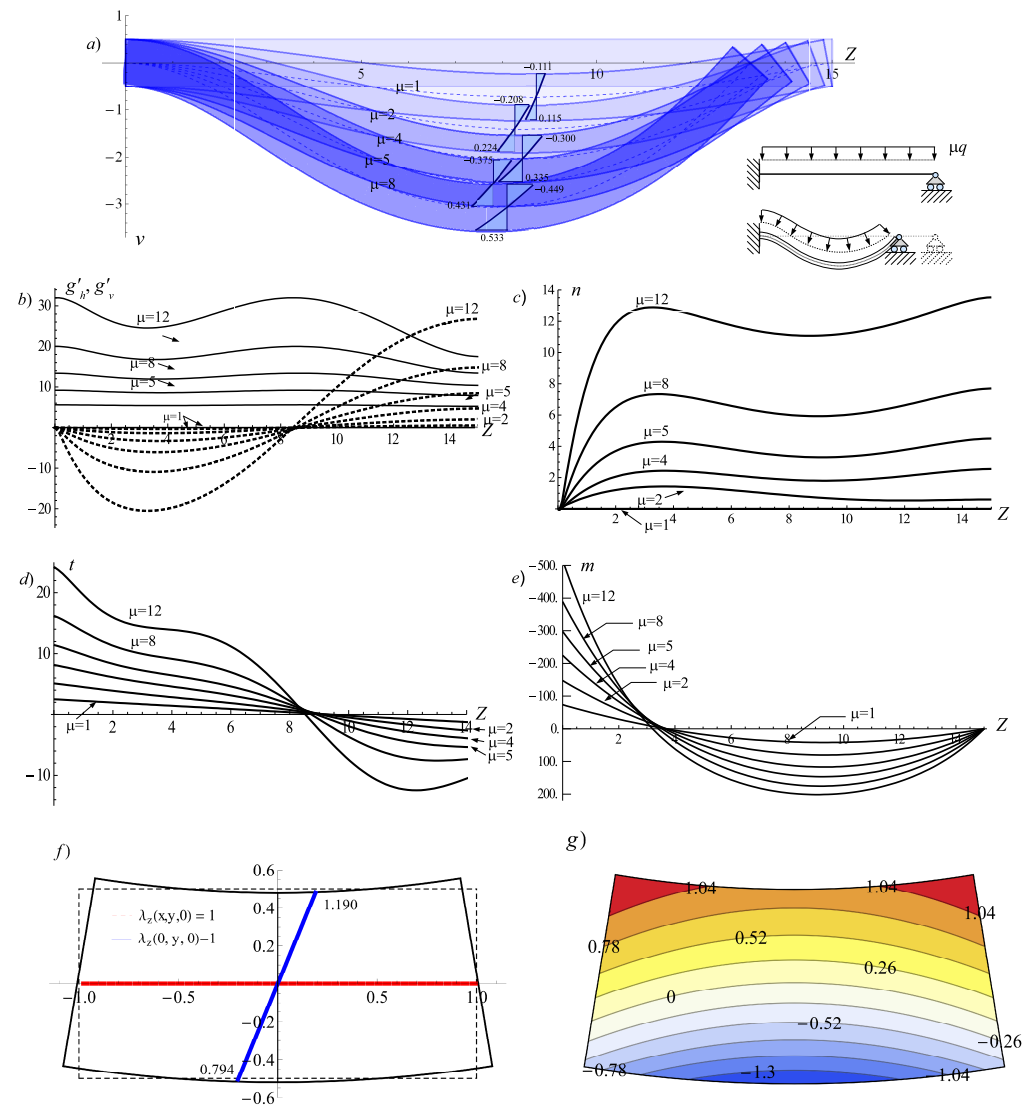

$g)$

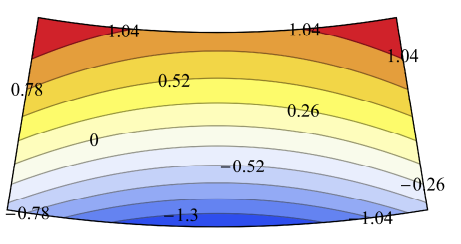

Figure 8: Beam clamped at one end and supported at the other under uniformly distributed vertical load with variable load multiplier $\mu$. Case with live loads. The loads act orthogonally to the deformed beam axis. a) Deflection curves. b) Intensity of the load density along the deformed beam axis. The vertical components are represented by the family of continuous lines, the horizontal components by the dotted lines. c) Normal force diagrams. d) Shear force diagrams. e) Bending moment diagrams. $f$ ) Eulerian stretches for the cross section with maximum bending moment. Vertical diagram of $\left(\lambda_{z}(0, y, 0)-1\right)$ and neutral axis for the deformation. $g$ ) Cauchy stress component $\mathrm{T}_{z}$ for the cross section with maximum bending moment. 


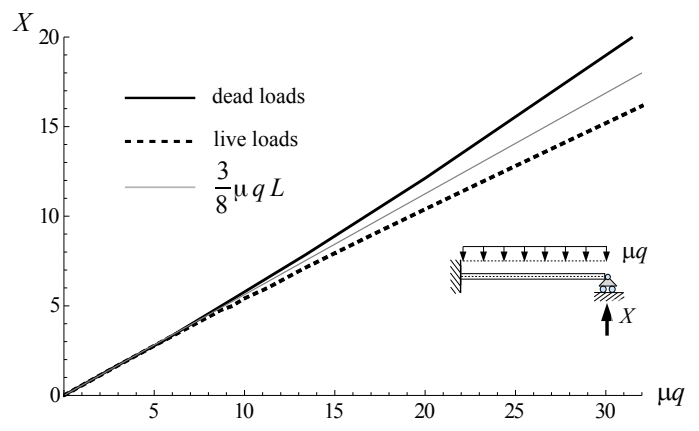

Figure 9: Vertical reaction at the right support versus the external loads $\mu q$. The intermediate thin line represents the corresponding values provided by the linear theory.

components of the load tend to balance each other. Moreover, with the growth of $\mu$, due to the inextensibility of the beam axis, the deflection curves tend to progressively approach one another. The shear force and bending moment diagrams do not show substantial changes with respect to the case of dead loads, while the normal force diagram is qualitatively different, not annulling more in the intermediate position.

Fig. 9 shows the values of the vertical reaction at the right support as the load multiplier increases. In the case of dead loads, these reactions are always greater than the corresponding reactions provided by the linear theory, $X=\frac{3}{8} \mu q L$, while in the case of live loads the reactions are always smaller.

The internal actions $n(s), t(s)$ and $m(s)$ are linked by the following differential equations:

$$
\begin{gathered}
-\frac{t}{R}+\frac{d n}{d s}+\mathbf{g}^{\prime} \cdot \mathbf{t}=0, \\
\frac{n}{R}+\frac{d t}{d s}+\mathbf{g}^{\prime} \cdot \mathbf{n}=0, \\
\frac{d m}{d s}-t=0,
\end{gathered}
$$

obtained from the equilibrium of a generic infinitesimal element of beam in the deformed configuration. The first equation derives from the translational equilibrium in the direction of $\mathbf{t}$, the second from the translational equilibrium in the direction $\mathbf{n}$ and the third from the rotational equilibrium. All functions involved into (18) depend on the curvilinear abscissa s. As discussed above, these equations in nonlinear theory are not sufficient to solve structures, but they can be however used a posteriori to check the correctness of the internal actions calculated before.

For more complex structures than those considered above, when for example the hypergeometric structure is composed of several beams, it is first necessary to identify the associated isogeometric structure and then replace the redundant 
constraints with their reactions. The number of subdomains, for which it is possible to describe the bending moment with a unique expression, is then estimated. For each single subdomain, the fundamental system (9) is imposed. Continuity conditions and compatibility equations at the suppressed constraints are then added. In this way, a coupled system of equations is written which must be entirely solved with the recursive numerical procedure. Obviously, the operational difficulties grow a lot with the number of subdomains.

\section{Numerical validation}

To estimate the accuracy of the solutions obtained for the bending of beams, the equilibrium equations will be used, checking that they are satisfied locally, that is for each single point of the beam. The axiom of force balance implies that the Piola-Kirchhoff stress tensor $\mathbf{T}_{R}$ must satisfy the following vectorial equation in the reference configuration:

$$
\operatorname{Div} \mathbf{T}_{R}+\mathbf{b}=\mathbf{o} .
$$

Substituting (3) into (19), calculating the material divergence of $\mathbf{T}_{R}$ and supposing that the body forces $\mathbf{b}$ are null, the following system of three partial differential equations is obtained:

$$
\left\{\begin{array}{l}
-S_{X} \frac{1}{r(s)} \sin \beta(s)+S_{X, X} \cos \beta(s)-S_{Y, Y} \sin \beta(s)=0 \\
S_{X} \frac{1}{r(s)} \cos \beta(s) \cos \theta(s)+S_{X, X} \sin \beta(s) \cos \theta(s)+S_{Y, Y} \cos \beta(s) \cos \theta(s) \\
-S_{Z} \frac{1}{R(s)} \cos \theta(s)-S_{Z, Z} \sin \theta(s)=0 \\
S_{X} \frac{1}{r(s)} \cos \beta(s) \sin \theta(s)+S_{X, X} \sin \beta(s) \sin \theta(s)+S_{Y, Y} \cos \beta(s) \sin \theta(s) \\
-S_{Z} \frac{1}{R(s)} \sin \theta(s)+S_{Z, Z} \cos \theta(s)=0 .
\end{array}\right.
$$

The expressions of the derivatives $S_{X, X}, S_{Y, Y}$ and $S_{Z, Z}$ are given in the Appendix. The first equation of the system (20) governs the equilibrium in the $X$ direction, the second along $Y$ and the third along the $Z$ axis of the undeformed beam.

The constitutive constants used in the applications of the previous two Sections have been made dimensionless, dividing them by the first constant $a$. If the elastic constants are without dimensions, then, stresses are also such. Similarly, the geometrical dimensions of the beam have been rendered dimensionless, dividing them by the height $\mathrm{H}$. In the same way, the variables $X, Y$ and $Z$ are normalized. Since these variables and stresses have been normalized, equilibrium equations (20) are also dimensionless, so that their comparison with the scalar zero takes full meaning. Evaluating therefore how much the three equations (20) deviate from zero, a measure of the accuracy of the solution obtained can be performed.

In [27], it has been shown that, for the points belonging to longitudinal basic line $X=0, Y=0$ and $Z=Z$, the equilibrium equations are exactly satisfied. For these points, the displacement field is correct being it kinematically 
compatible and equilibrated. When leaving the basic line, equilibrium equations are no longer so well satisfied and approximations grow as the edges of the cross sections are approached. This is because for these points the hypotheses underlying the kinematic model are less accurate. However, as a result of the continuity of the displacement field, it is reasonable to expect a central core of the cross sections, where the solution is still acceptable since the equilibrium equations are close to zero. The extension of this central core increases with the length of the beam, i.e. in the case of slender beams.

Given the above, a numerical analysis is performed below, evaluating the equations (20) in all the points of some discrete cross sections of the examples carried out in the previous Sections. The results of computations are delivered in two-dimensional diagrams with the shape similar to the cross section of the beam. In these diagrams some contour lines, which join the points where the equations (20) give the same numeral values, are shown.

Fig. 10 summarizes the results obtained for the cantilever beam of Fig. 3, with the maximum load multiplier $\mu=30$. Equilibrium equations (20) have been evaluated numerically in the three distinct cross sections: $s=0, s=L / 3$ and $s=2 L / 3$. The three images arranged on a line of Fig. 10 are relative to the equilibrium for the same cross section along the $X, Y$ and $Z$ axis, respectively. As shown in Figs. 10a, 10d and $10 \mathrm{~g}$ there is a wide vertical band in the central position where the equilibrium in the $X$ direction is practically satisfied. The approximations grow by moving towards the left and right edge of the cross sections. For equilibria along the $Y$ axis (cf. Figs. 10b, 10e and 10h) and along the $Z$ axis (cf. Figs. 10c, 10f and 10i), the band with practically zero equations is horizontal. In these cases, approximations grow moving towards the upper and lower edge. As can be observed from the nine images of Fig. 10, the maximum deviations from the zero, which occur at the edges of the cross section, are really very modest. In the most penalizing situation, that is at the upper and lower edges of the cross section at the clamped end, the equilibrium along the $Y$ axis shows an approximation of just 1\%. And this occurs for the cantilever beam subjected to a severe loading condition, capable of producing high curvatures.

In the same way, the numerical analysis was performed for the hypergeometric scheme of a beam clamped at one end and supported at the other, illustrated by Fig. 7. To have a bending moment at the clamped end comparable to that of the previous case, the load multiplier $\mu$ has been chosen equal to six. The results obtained are summarized in Fig. 11. As can be noted from this last figure, all deviations are smaller than in the previous example. This is due to the fact that the hypergeometric scheme considered is less deformable than the above cantilever beam, so it experiences smaller curvatures.

The numerical analyzes carried out in this Section show that the proposed solution for the bending of nonlinear beams is very reliable. In general, the accuracy of the solution grows when increasingly slender beams are considered, in the sense that the equilibrium equations are practically satisfied in every point of the beam. 

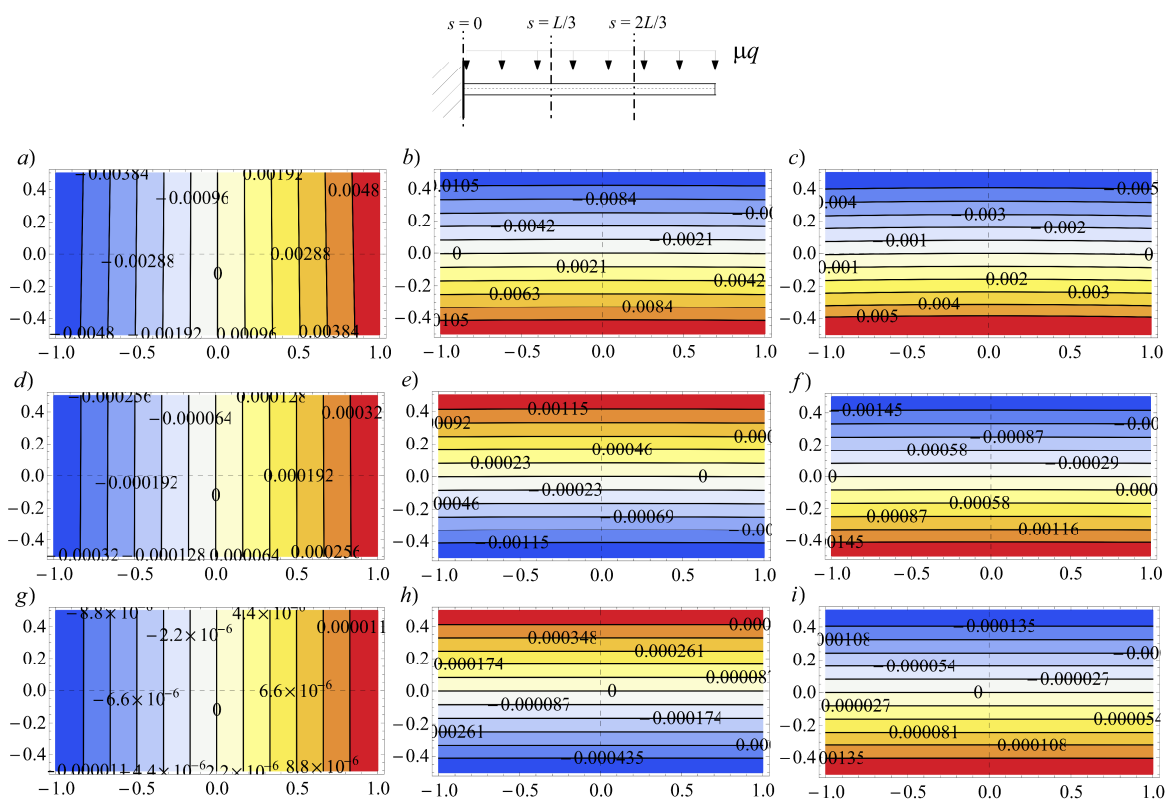

Figure 10: Plot of the equilibrium equations (20) evaluated locally in every point of some discrete cross sections of the cantilever beam illustrated by Fig. 3. Case with $\mu=30$. (a), (b) and (c) Equilibrium along the $X, Y$ and $Z$ axis at the cross section $s=0$, for which $m_{x}=-384.38, \theta=0, R=25.628, R^{(1)}=0.618$. (d), (e) and (f) Equilibrium along the $X, Y$ and $Z$ axis at the cross section $s=L / 3$, for which $m_{x}=-99.52, \theta=1.0619, R=-98.985, R^{(1)}=3.024$. (g), (h) and (i) Equilibrium along the $X, Y$ and $Z$ axis at the cross section $s=2 L / 3$, for which $m_{x}=-18.53, \theta=1.317, R=-531.62, R^{(1)}=23.28$. 

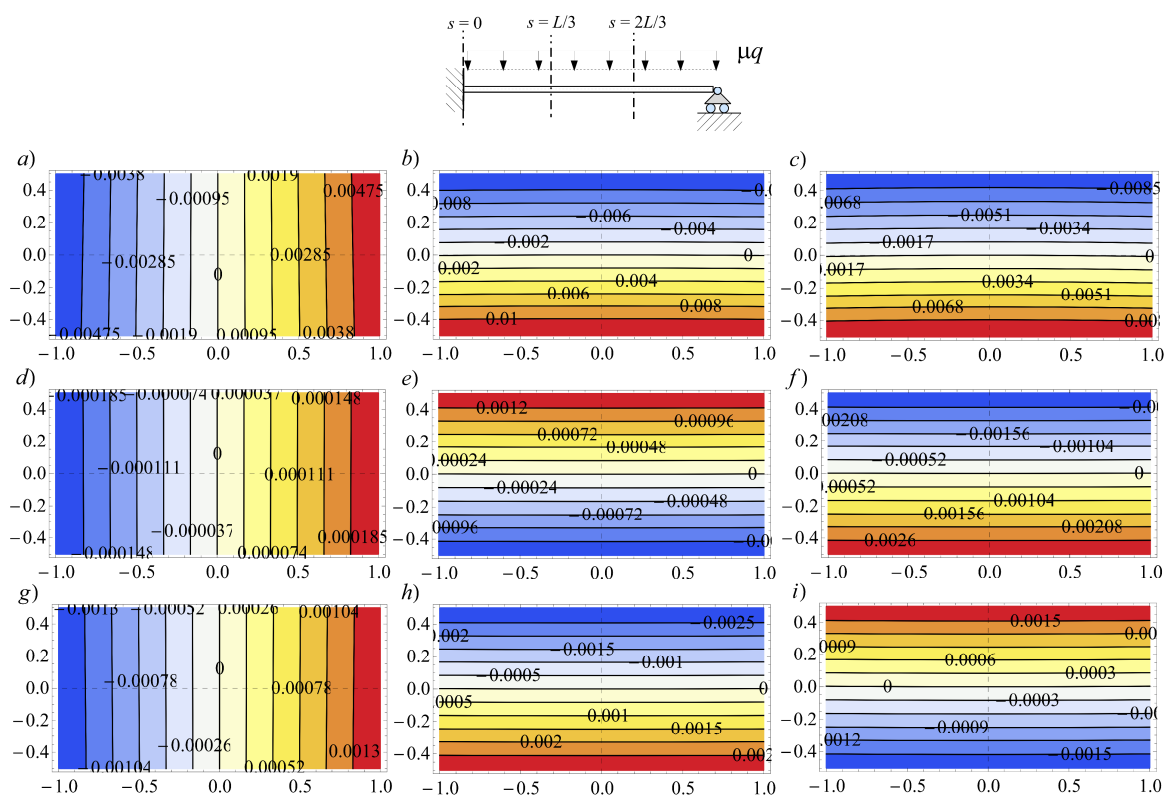

Figure 11: Plot of the equilibrium equations (20) evaluated locally in every point of some discrete cross sections of the beam clamped at one end and supported at the other illustrated by Fig. 7. Case with $\mu=6$ (a), (b) and (c) Equilibrium along the $X, Y$ and $Z$ axis at the cross section $s=0$, for which $m_{x}=-383.446, \theta=0, R=25.691 R^{(1)}=1.025$. (d), (e) and (f) Equilibrium along the $X, Y$ and $Z$ axis at the cross section $s=L / 3$, for which $m_{x}=75.926, \theta=0.575, R=-129.744, R^{(1)}=8.600$. (g), (h) and (i) Equilibrium along the $X, Y$ and $Z$ axis at the cross section $s=2 L / 3$, for which $m_{x}=200.63, \theta=-0.274, R=-49.101, R^{(1)}=-0.331$. 


\section{Conclusions}

In this paper, the equilibrium problem of high-flexible structures has been investigated. The analysis is based on a recent solution for the bending of nonlinear beams obtained by Lanzoni and Tarantino [1] in the context of finite elasticity. In detail, this solution was obtained without introducing any hypothesis of smallness for displacement and deformation fields, adopting a nonlinear constitutive law and considering the complete three-dimensionality of the beam. Using this basic solution, the mechanics of high-flexible structures, without simplifications and linearizations, has been formulated, being able to fully capture the nonlinear aspects. External static loads dependent on the deformation are also considered (live loads) and for them a mathematical description has been performed to facilitate their prescription in the problem formulation.

In nonlinear theory, it is not possible to solve a statically determined structure using only the equilibrium static equations. In fact, both the statically determined and the indeterminate structures must be solved by imposing the conditions of equilibrium with respect to the deformed configuration. The deformed configuration is not known a priori, but it must be determined using compatibility conditions. In the context of nonlinear mechanics this suggests abandoning the classical classification of structures dictated by the static point of view in favor of a more coherent classification based on the kinematic aspects. Thus, on the basis of the degree of kinematic constraints, a classification in isogeometric and hypergeometric structures has been proposed.

After dealing with the general mechanical features of the class of highflexible structures, for the isogeometric beams, the governing equations, whose unknowns are the displacement components and the rotations along the deformed beam axis, are derived. These governing equations assume the form of a highly nonlinear coupled system of three equations in integral form. This system is solved by means of an iterative numerical procedure. This initial computation allows to trace the deflection curves and to determine the internal actions (i.e. normal force, shear force and bending moment) along these curves. Subsequently, the functions that describe the longitudinal and transverse curvature radii are evaluated. With these two functions, the displacement of each point of the beam can be computed, thus obtaining the three-dimensional shape assumed by the beam in the deformed configuration. Finally, the analysis of stretches and stresses for each cross sections can be carried out, using both the Lagrangian and Eulerian description.

The exposed analysis for isogeometric beams has been then applied to two typical structural systems: The cantilever beam and the simply supported beam. Both the beams are subjected to dead and live uniformly distributed loads. As the load multiplier grows, the results have been shown by a series of graphs.

The equilibrium problem for the hypergeometric beams has been formulated by adding to the governing equations of the isogeometric beams the compatibility conditions at the redundant constraints. Differently from the linear theory, these additional compatibility conditions must be imposed in the deformed configuration. In the case of hypergeometric beams, the bending moment depends 
also on the redundant reactions. The system of governing equations so obtained is solved again with an iterative numeral procedure.

As example of application, a beam clamped at one end and supported at the other, subjected to both dead and live loads, is considered. The results are discussed and shown in some graphs.

The accuracy of the solutions obtained was estimated by evaluating how the equilibrium equations, appropriately made dimensionless, deviate from the zero. The numerical analysis performed in last Section showed that these deviations are practically zero in a central core of the cross sections and very close to zero in the points outside such core.

\section{Acknowledgement}

Financial support from the Italian Ministry of Education, University and Research (MIUR) in the framework of the Project PRIN "Modelling of constitutive laws for traditional and innovative building materials" (code 2017HFPKZY) is gratefully acknowledged.

\section{Appendix: Expressions of derivatives $S_{X, X}, S_{Y, Y}$ and $S_{Z, Z}$}

The derivatives of the functions $S_{J}=2\left(\omega_{1}+I_{1} \omega_{2}\right) \lambda_{J}-2 \omega_{2} \lambda_{J}^{3}+2 I_{3} \omega_{3} \frac{1}{\lambda_{J}}$, with $J=X, Y$ and $Z$, contained in the system (20), have the following expressions:

$$
\begin{aligned}
& S_{X, X}=2\left(\omega_{1, X}+I_{1, X} \omega_{2}+I_{1} \omega_{2, X}\right) \lambda_{X}-2 \omega_{2, X} \lambda_{X}^{3}+2\left(I_{3, X} \omega_{3}+I_{3} \omega_{3, X}\right) \frac{1}{\lambda_{X}}, \\
& S_{Y, Y}=2\left(\omega_{1, Y}+I_{1, Y} \omega_{2}+I_{1} \omega_{2, Y}\right) \lambda_{Y}+2\left(\omega_{1}+I_{1} \omega_{2}\right) \lambda_{Y, Y}-2 \omega_{2, Y} \lambda_{Y}^{3} \\
& -6 \omega_{2} \lambda_{Y}^{2} \lambda_{Y, Y}+2 I_{3, Y} \omega_{3} \frac{1}{\lambda_{Y}}+2 I_{3} \omega_{3, Y} \frac{1}{\lambda_{Y}}-2 I_{3} \omega_{3} \frac{\lambda_{Y Y}^{2}}{\lambda_{Y}^{2}}, \\
& S_{Z, Z}=2\left(\omega_{1, Z}+I_{1, Z} \omega_{2}+I_{1} \omega_{2, Z}\right) \lambda_{Z}+2\left(\omega_{1}+I_{1} \omega_{2}\right) \lambda_{Z, Z}-2 \omega_{2, Z} \lambda_{Z}^{3} \\
& -6 \omega_{2} \lambda_{Z}^{2} \lambda_{Z, Z}+2 I_{3, Z} \omega_{3} \frac{1}{\lambda_{Z}}+2 I_{3} \omega_{3, Z} \frac{1}{\lambda_{Z}}-2 I_{3} \omega_{3} \frac{\lambda_{Z, Z}}{\lambda_{Z}^{2}},
\end{aligned}
$$

with $\omega_{i}=\frac{\partial \omega}{\partial I_{i}}, I_{i, K}=\frac{\partial I_{i}}{\partial K}$ and $\omega_{i, K}=\frac{\partial}{\partial K}\left(\frac{\partial \omega}{\partial I_{i}}\right)$ for $i=1,2,3, K=X, Y$ and $Z$. On the basis of the kinematic model, the following quantities can be computed:

$$
\begin{aligned}
& \lambda_{Y, Y}=\frac{\partial \lambda_{Y}}{\partial Y}=-\frac{1}{r(s)} e^{-\frac{Y}{r(s)}} \\
& \lambda_{Z, Z}=\frac{\partial \lambda_{Z}}{\partial Z}=r(s)\left[1-e^{-\frac{Y}{r(s)}} \cos \beta(s)\right] \theta^{(2)}(s), \text { with } \frac{1}{R(s)}=\theta^{(1)}(s), \\
& I_{1}=2 e^{-\frac{2 Y}{r(s)}}+\left[1+\frac{r(s)}{R(s)}\left(1-e^{-\frac{Y}{r(s)}} \cos \beta(s)\right)\right]^{2}, \\
& I_{3}=e^{-\frac{4 Y}{r(s)}}\left[1+\frac{r(s)}{R(s)}\left(1-e^{-\frac{Y}{r(s)}} \cos \beta(s)\right)\right]^{2} \\
& I_{1, X}=2 \lambda_{Z} \frac{1}{R(s)} e^{-\frac{Y}{r(s)}} \sin \beta(s),
\end{aligned}
$$




$$
\begin{aligned}
& I_{1, Y}=-\frac{4}{r(s)} e^{-\frac{2 Y}{r(s)}}+2 \lambda_{Z} \frac{1}{R(s)} e^{-\frac{Y}{r(s)}} \cos \beta(s), \\
& I_{1, Z}=2 \lambda_{Z} r(s)\left(1-e^{-\frac{Y}{r(s)}} \cos \beta(s)\right) \theta^{(2)}(s), \\
& I_{3, X}=2 e^{-\frac{5 Y}{r(s)}} \lambda_{Z} \frac{1}{R(s)} \sin \beta(s), \\
& I_{3, Y}=-\frac{4}{r(s)} e^{-\frac{4 Y}{r(s)}} \lambda_{Z}^{2}+2 e^{-\frac{5 Y}{r(s)}} \lambda_{Z} \frac{1}{R(s)} \cos \beta(s), \\
& I_{3, Z}=2 e^{-\frac{4 Y}{r(s)}} \lambda_{Z} r(s)\left(1-e^{-\frac{Y}{r(s)}} \cos \beta(s)\right) \theta^{(2)}(s) .
\end{aligned}
$$

For a Mooney-Rivlin material the following derivatives, contained in the previous equations, are calculated:

$$
\begin{aligned}
& \omega_{1}=a, \quad \omega_{1, X}=\omega_{1, Y}=\omega_{1, Z}=0, \\
& \omega_{2}=b, \quad \omega_{2, X}=\omega_{2, Y}=\omega_{2, Z}=0, \\
& \omega_{3}=c-\frac{d}{2 I_{3}}, \\
& \omega_{3, X}=\frac{d}{R(s)} \frac{1}{\lambda_{Z}^{3}} e^{\frac{3 Y}{r(s)}} \sin \beta(s), \\
& \omega_{3, Y}=-\frac{d}{2} e^{\frac{4 Y}{r(s)}}\left(\frac{4}{r(s)} \frac{1}{\lambda_{Z}^{2}}-\frac{2}{R(s)} \frac{1}{\lambda_{Z}^{3}} e^{\left.-\frac{Y}{r(s)} \cos \beta(s)\right),}\right. \\
& \omega_{3, Z}=d e^{\frac{4 Y}{r(s)}} \frac{1}{\lambda_{Z}^{3}} r(s)\left(1-e^{-\frac{Y}{r(s)}} \cos \beta(s)\right) \theta^{(2)}(s) .
\end{aligned}
$$

\section{References}

[1] L. Lanzoni, A.M. Tarantino, The bending of beams in finite elasticity. $J$. Elasticity (2019) DOI: 10.1007/s10659-019-09746-8.

[2] Jacob Bernoulli, Specimen alterum calculi differentialis in dimetienda spirali logarithmica, loxodromiis nautarum et areis triangulorum sphaericorum. Una cum additamento quodam ad problema funicularium, aliisque. Acta Eruditorum, Junii (1691) 282-290 - Opera, 442-453.

[3] Daniel Bernoulli. The 26th letter to Euler (1742). Correspondance mathématique et physique de quelques célèbres géomètres du XVIIIème siècle, Tome 2, P. H. Fuss, St. Pétersbourg (1843).

[4] L. Euler, Additamentum I de curvis elasticis, methodus inveniendi lineas curvas maximi minimivi proprietate gaudentes. Bousquent, Lausanne (1744).

[5] L. Euler, Genuina principia doctrinae de statu aequilibrii et motu corporum tam perfecte flexibilium quam elasticorum. Opera Omnia II 11 (1771) $37-61$.

[6] J. L. de Lagrange, Sur la figure des colonnes. Miscellanea Taurinensia 5 (1770-1773) 125-170. 
[7] M. Born, Untersuchungen über die Stabilität der elastischen Linie in Ebene und Raum, under verschiedenen Grenzbedingungen. PhD thesis, University of Göttingen, Germany (1906).

[8] H.J. Barten, On the deflection of a cantilever beam. Q. Appl. Math. 2 (1944) 168-171.

[9] H.J. Barten, On the deflection of a cantilever beam. Q. Appl. Math. 3 (1945) 275-276.

[10] K.E. Bisshopf, D.C. Drucker, Large deflection of cantilever beams. Q. Appl. Math. 3 (1945) 272-275.

[11] F.V. Rohde, Large deflections of a cantilever beam with uniformly distributed load. Q. Appl. Math. 11 (1953) 337-338.

[12] R. Frisch-Fay, Flexible bars. Butterworths, London (1962).

[13] J. Wang, J.K. Chen and S. Liao, An explicit solution of the large deformation of a cantilever beam under point load at the free tip. J. Comp. Appl. Math. 212 (2008) 320-330.

[14] T.M. Wang, S.L. Lee, O.C. Zienkiewicz, Numerical analysis of large deflections of beams. Inter. J. Mech. Scien. 3 (1961) 219-228.

[15] T.M. Wang, Non-linear bending of beams with uniformly distributed loads. Inter. J. Nonlinear Mech. 4 (1969) 389-395.

[16] C. Armanini, F. Dal Corso, D. Misseroni and D. Bigoni, From the elastica compass to the elastica catapult: an essay on the mechanics of soft robot arm. Proc. of the Royal Soc. A 473 (2017) doi.org/10.1098/rspa.2016.0870.

[17] A. Della Corte, F. dell'Isola, R. Esposito and M. Pulvirenti, Equilibria of a clamped Euler beam (Elastica) with distributed load: Large deformations. Math. Models and Methods in Appl. Sciences 27 (2017) 1391-1421.

[18] M.P. Silverman, J. Farrah, Bending of a Tapered Rod: Modern Application and Experimental Test of Elastica Theory. World Journal of Mechanics $\mathbf{8}$ (2018) 272-300.

[19] M. Batista, Large deflections of an elastic rod in contact with a flat wall. Inter. J. Solids Struct. 115-116 (2017) 53-60.

[20] A. Cazzolli, D. Misseroni, F. Del Corso, Elastica catastrophe machine: theory, design and experiments. J. Mech. Phys. Solids (2019) in press. doi.org/10.1016/j.jmps.2019.103735.

[21] H. Elettro, P. Grandgeorge, S. Neukirch, Elastocapillary coiling of an elastic rod inside a drop. J. Elasticity 127 (2017) 235-247. 
[22] A. Liakou, Application of optimal control method in buckling analysis of constrained elastica problems. Inter. J. Solids Struct. 141-142 (2018) 158172 .

[23] T.G. Sano, H. Wada, Snap-buckling in asymmetrically constrained elastic strips. Phys. Rev. E 97 (2018) 013002.

[24] H. Singh, J.A. Hanna, On the planar Elastica, stress, and material stress. J. Elasticity 136 (2019) 87-101.

[25] D. Bigoni, F. Bosi, D. Misseroni, F. Del Corso, G. Novelli, New phenomena in nonlinear elastic structures: from tensile buckling to configurational forces, in CISM Lecture Notes No. 562 "Extremely Deformable Structures" (2015) edited by D. Bigoni, Springer, ISBN 978-3-7091-1876-4.

[26] O.M. O'Reilly, Modeling nonlinear problems in the mechanics of strings and rod. Springer (2017).

[27] L. Lanzoni, A.M. Tarantino, Finite anticlastic bending of hyperelastic solids and beams. J. Elasticity 131 (2018) 137-170. https://doi.org/10.1007/s10659-017-9649-y.

[28] R.S. Rivlin, Large elastic deformations of isotropic materials. V. The problem of flexure. Proc. R. Soc. Lond. A 195 (1949) 463-473.

[29] F.O. Falope, L. Lanzoni, A.M. Tarantino, The bending of fully nonlinear beams. Theoretical, numerical and experimental analyses. Inter. J. Eng. Scien. 145 (2019) 103167.

[30] F.O. Falope, L. Lanzoni, A.M. Tarantino, Bending device and anticlastic surface measurement of solids under large deformations and displacements. Mech. Res. Comm. 97 (2019) 52-56.

[31] A.M. Tarantino, L. Lanzoni, F.O. Falope, The bending theory of fully nonlinear beams. Springer (2019)

[32] A.M. Tarantino, Crack propagation in finite elastodynamics. Math. Mech. of Solids 10 (2005) 577-601.

[33] A.M. Tarantino, Equilibrium paths of a hyperelastic body under progressive damage. J. Elasticity 114 (2014) 225-250.

[34] L. Lanzoni, A.M. Tarantino, Damaged hyperelastic membranes. Inter. J. Nonlinear Mech. 60 (2014) 9-22.

[35] L. Lanzoni, A.M. Tarantino, Equilibrium configurations and stability of a damaged body under uniaxial tractions. ZAMP J. of Appl. Math. and Phys. 66 (2015) 171-190. 
[36] L. Lanzoni, A.M. Tarantino, A simple nonlinear model to simulate the localized necking and neck propagation. Inter. J. Nonlinear Mech. 84 (2016) 94-104.

[37] M. Pelliciari, A.M. Tarantino, Equilibrium paths for Von Mises trusses in finite elasticity. J. Elasticity (2019) in press. DOI: 07/s10659-019-09731-1.

[38] M. Pelliciari, A.M. Tarantino, Equilibrium paths of a three-bar truss in finite elasticity with an application to graphene. Math. Mech. of Solids (2019) in press. https://doi.org/10.1177/1081286519887470. 